Proceedings of the Edinburgh Mathematical Society (2008) 51, 171-199 (C)

DOI:10.1017/S001309150500177X Printed in the United Kingdom

\title{
AN ANALYTICAL APPROACH TO HEAT KERNEL ESTIMATES ON STRONGLY RECURRENT METRIC SPACES
}

\author{
JIAXIN HU \\ Department of Mathematical Sciences, Tsinghua University, Beijing 100084, \\ People's Republic of China (hujiaxin@mail.tsinghua.edu.cn)
}

(Received 5 December 2005)

\begin{abstract}
In this paper we prove that sub-Gaussian estimates of heat kernels of regular Dirichlet forms are equivalent to the regularity of measures, two-sided bounds of effective resistances and the locality of semigroups, on strongly recurrent compact metric spaces. Upper bounds of effective resistances imply the compact embedding theorem for domains of Dirichlet forms, and give rise to the existence of Green functions with zero Dirichlet boundary conditions. Green functions play an important role in our analysis. Our emphasis in this paper is on the analytic aspects of deriving two-sided sub-Gaussian bounds of heat kernels. We also give the probabilistic interpretation for each of the main analytic steps.
\end{abstract}

Keywords: heat kernel; effective resistance; $\alpha$-set; Dirichlet form; Green function

2000 Mathematics subject classification: Primary 32W05

Secondary $35 \mathrm{R} 20$

\section{Introduction}

In recent years, people have studied heat kernels or transition densities on fractals, and have obtained elegant sub-Gaussian estimates of the form

$$
\begin{aligned}
a_{1} t^{-\alpha / \beta} \exp \left(-b_{1}\left(t^{-1 / \beta} d(x, y)\right)^{\gamma_{1}}\right) & \leqslant p(t, x, y) \\
& \leqslant a_{2} t^{-\alpha / \beta} \exp \left(-b_{2}\left(t^{-1 / \beta} d(x, y)\right)^{\gamma_{2}}\right)
\end{aligned}
$$

for heat kernels $p(t, x, y)$ on a certain class of fractals, where $a_{i}, b_{i}, \gamma_{i}>0$ for $i=1,2$ and $\alpha>0, \beta \geqslant 2$, and $d(x, y)$ is a metric on the fractal considered (see, for example, $[\mathbf{2 - 4}, \mathbf{1 1}, \mathbf{1 7}])$. In order to obtain (1.1), the theory of Markov processes has been used extensively in the literature cited above. On the other hand, people are also interested in obtaining (1.1) in a purely analytic approach, without recourse to the theory of Markov processes. Recall that, for the classical case where $\beta=2$, there have been analytic approaches to deriving (1.1) (see, for example, $[\mathbf{1}, \mathbf{6}, \mathbf{8}]$ ). However, to our knowledge, no analytic approach is available for the fractal case or, more generally, for metric spaces where $\beta>2$. Note that the existent analytic method for $\beta=2$ is not applicable to the case where $\beta>2$. 
In this paper we will establish analytically sub-Gaussian estimates of the type (1.1) for heat kernels on compact metric spaces satisfying the chain condition. More precisely, we show that sub-Gaussian estimates for heat kernels of Dirichlet forms on strongly recurrent compact metric spaces are equivalent to the regularity of measures, polynomial growth of effective resistances and the locality of the semigroups (see Theorem 2.2). We explain here how to derive sub-Gaussian estimates of heat kernels analytically. Let $X$ be a metric space, and let $B(x, r)$ be an open ball in $X$. The effective resistance $R\left(x, B(x, r)^{\mathrm{c}}\right)$ between $x \in X$ and $B(x, r)^{\mathrm{c}}$ is well defined. This gives rise to the existence of the Green function $g_{B(x, r)^{\mathrm{c}}}^{x}(\cdot)$ vanishing on $B(x, r)^{\mathrm{c}}$. The Green function $g_{B(x, r)^{\mathrm{c}}}^{x}(y)$ has the same polynomial growth as the effective resistance $R\left(x, B(x, r)^{\mathrm{c}}\right)$, for $x$ and $y$ sufficiently close. An important step is to bound from above the solution of the linear heat equation with initial values vanishing on the ball (see (5.55), below). This can be achieved by estimating the solution $u_{\lambda}$ to a linear elliptic equation (see (5.52), below) for $\lambda>0$ (see the crucial estimate (5.48)). We obtain (5.48) by applying the locality of the semigroup of the Dirichlet form, together with the estimate of the solution $u_{0}$ to a Poisson equation with zero boundary condition (see (5.20), (5.21), below). We estimate $u_{0}$ by using the regularity of the measure and the estimate of the Green function. Once we obtain the estimate (5.55), off-diagonal upper bounds of heat kernels easily follow by using on-diagonal upper bounds. Lower off-diagonal bounds of heat kernels are derived more easily by using the chain argument (see, for example, $[\mathbf{2}, \mathbf{1 6}]$ ).

We mention in passing here that there is a probabilistic interpretation for each of the main analytic steps for deriving (5.55), which will be pointed out in the remarks following it. (The reader may consult $[\mathbf{2}, \mathbf{1 3}]$ for the probabilistic arguments.) A result similar to Theorem 2.2 was obtained on strongly recurrent graphs in [5] and on resistance forms in $[\mathbf{2 0}]$, by using the probability theory.

Finally, we remark that the method of this paper could be applicable to more general bounded or unbounded metric spaces. We will address this issue elsewhere.

\section{Notation.}

In the following, we keep $c_{i}, \varepsilon_{i}, i \geqslant 0$, fixed, and use $c, c^{\prime}$ and $c^{\prime \prime}$ to denote general constants. For two functions $f$ and $g$, by $f \asymp g$ we mean that there is some $c>0$ such that $c^{-1} f \leqslant g \leqslant c f$.

\section{Preliminaries and main results}

Let $(X, d)$ be a compact metric space satisfying the chain condition, that is, there exists a constant $c_{0}>0$ such that, for any distinct points $x, y \in X$ and any integer $n \geqslant 1$, there exists a sequence of points $\left\{x_{k}\right\}_{k=0}^{n}$ in $X$ such that $x_{0}=x, x_{n}=y$ and

$$
d\left(x_{i}, x_{i+1}\right) \leqslant c_{0} n^{-1} d(x, y), \quad 0 \leqslant i \leqslant n-1 .
$$

Set $r_{0}:=\operatorname{diam}(X)<\infty$, the diameter of $X$. Let $B(x, r)=\{y \in X: d(y, x)<r\}$ be an open ball in $X$ with centre $x$ and radius $r$. Denote by $\partial B(x, r)=\{y \in X: d(y, x)=r\}$ 
the boundary of the ball $B(x, r)$. For any $x \in X$ and $r \in\left(0, r_{0}\right)$, the chain condition and the compactness of $X$ imply that the boundary $\partial B(x, r)$ of any ball $B(x, r)$ is not empty.

Let $\mu$ be a Borel measure with $\operatorname{supp} \mu=X$. For simplicity, we assume that $\mu(X)=1$. For $\alpha>0$, we say that $\mu$ satisfies condition (A1) if $\mu$ is $\alpha$-regular, that is, there exists some $c_{1}>0$ such that

$$
c_{1}^{-1} r^{\alpha} \leqslant \mu(B(x, r)) \leqslant c_{1} r^{\alpha}
$$

for all $x \in X$ and all $0<r \leqslant r_{0}$. We call $X$ an $\alpha$-set if (A1) holds.

For $1 \leqslant p<\infty$, denote by $L^{p}(\mu):=L^{p}(X, d, \mu)$ the space of all $p$-integrable real-valued functions on $X$ with the norm

$$
\|u\|_{p}:=\left(\int_{X}|u(x)|^{p} \mathrm{~d} \mu(x)\right)^{1 / p} .
$$

Denote by $C(X)$ (or $C_{0}(X)$ ) the space of all continuous functions (or all continuous functions with compact support) on $X$ with uniform norm. Let $(\mathcal{E}, \mathcal{F})$ be a Dirichlet form on $L^{2}(\mu)$. Recall that $(\mathcal{E}, \mathcal{F})$ is regular if $\mathcal{F} \cap C_{0}(X)$ is dense in $\mathcal{F}$ with norm

$$
\left(\mathcal{E}(u)+\|u\|_{2}^{2}\right)^{1 / 2}
$$

and dense in $C_{0}(X)$ with uniform norm. (Here we use the abbreviation $\mathcal{E}(u):=\mathcal{E}(u, u)$.) The form $(\mathcal{E}, \mathcal{F})$ is local if $\mathcal{E}(u, v)=0$ for $u, v \in \mathcal{F}$ with disjoint supports, and irreducible if $\mathcal{E}(u)=0$ when and only when $u$ is constant $[\mathbf{1 2}]$. Let $\left\{T_{t}\right\}_{t \geqslant 0}$ be the semigroup associated with $(\mathcal{E}, \mathcal{F})$. For any $t>0$, the operator $\left\{T_{t}\right\}_{t \geqslant 0}$ may possess an integral kernel $p(t, x, y)$, termed the heat kernel, that is, for $t>0$ and $\mu$-almost all $x \in X$,

$$
T_{t} u(x)=\int_{X} u(y) p(t, x, y) \mathrm{d} \mu(y)
$$

for $u \in L^{2}(\mu)$. Since $\mathcal{E}(1)=0$, we see that $T_{t} 1=1$ (see [2, Lemma 4.10, p. 50]). Therefore, if the heat kernel $p(t, x, y)$ exists, for $t>0$ and $\mu$-almost all $x \in X$, we have

$$
\int_{X} p(t, x, y) \mathrm{d} \mu(y)=1 .
$$

Note that $\left\{T_{t}\right\}_{t \geqslant 0}$ is strongly continuous in the $L^{2}(\mu)$-norm, that is

$$
\lim _{t \rightarrow 0}\left\|T_{t} u-u\right\|_{2}=0, \quad u \in L^{2}(\mu)
$$

Let $H$ be the infinitesimal generator of $\left\{T_{t}\right\}_{t \geqslant 0}$ in the $L^{2}(\mu)$-norm, that is

$$
\lim _{t \rightarrow 0}\left\|t^{-1}\left(T_{t} u-u\right)-H u\right\|_{2}=0
$$

for $u \in \mathcal{D}(H)$, the space of all functions $u \in L^{2}(\mu)$ such that the above limit exists for some function $H u \in L^{2}(\mu)$. Note that $\mathcal{D}(H)$ is dense in $L^{2}(\mu)$. We shall see below that the semigroup $\left\{T_{t}\right\}$ considered in this paper is actually the Feller semigroup, that is, $T_{t} f \geqslant 0$ for $f \geqslant 0, T_{t} f \leqslant 1$ for $f \leqslant 1$ and

(i) for any $t>0$, the operator $T_{t}: C(X) \rightarrow C(X)$, and

(ii) $\left\|T_{t} u-u\right\|_{C(X)} \rightarrow 0$ as $t \rightarrow 0$ for $u \in C(X)$. 
For a Feller semigroup $\left\{T_{t}\right\}$ and $u \in C(X)$, if there is a function $v \in C(X)$ such that

$$
\lim _{t \rightarrow 0}\left\|t^{-1}\left(T_{t} u-u\right)-v\right\|_{C(X)}=0
$$

then we define

$$
\Delta u(x)=v(x), \quad x \in X .
$$

Let $\mathcal{D}(\Delta)$ be the domain of $\Delta$. Clearly, (2.6) implies that

$$
\lim _{t \rightarrow 0} t^{-1}\left(T_{t} u(x)-u(x)\right)=v(x)
$$

for each point $x \in X$. Note that $H$ is the extension of the linear operator $\Delta: \mathcal{D}(\Delta) \rightarrow$ $C(X)$. In the following, we will use the fact that

$$
\mathcal{E}(u, v)=\lim _{t \rightarrow 0} \mathcal{E}_{t}(u, v):=\lim _{t \rightarrow 0}\left(\frac{u-T_{t} u}{t}, v\right), \quad u, v \in \mathcal{F},
$$

where $(\cdot, \cdot)$ is the inner product in $L^{2}(\mu)$ (see [12, Lemma 1.3.4, p. 22]).

For any non-empty open subset $D$ of $X$, let

$$
\mathcal{F}_{D}:=\left\{u \in \mathcal{F}:\left.u\right|_{D^{c}}=0\right\},
$$

where $D^{\mathrm{c}}=X \backslash D$. If $(\mathcal{E}, \mathcal{F})$ is a regular Dirichlet form, then $\left(\mathcal{E}, \mathcal{F}_{D}\right)$ is also a regular Dirichlet form $[\mathbf{1 2}$, Theorem 4.4.3, p. 154]. As in the case of the form $(\mathcal{E}, \mathcal{F})$, we denote by $\left\{T_{t}^{D}\right\}_{t \geqslant 0}$ and $H_{D}$ the semigroup and generator for the form $\left(\mathcal{E}, \mathcal{F}_{D}\right)$, respectively. In particular, denote by $p_{D}(t, x, y)$ the heat kernel of $\left(\mathcal{E}, \mathcal{F}_{D}\right)$, if it exists. We extend $p_{D}(t, x, y)$ so that $p_{D}(t, x, y)=0$ for $t>0$ if $x \in D^{\mathrm{c}}$ or $y \in D^{\mathrm{c}}$. For any $u, v \in \mathcal{F}_{D} \subset \mathcal{F}$, observe that

$$
\lim _{t \rightarrow 0}\left(\frac{u-T_{t} u}{t}, v\right)=\mathcal{E}(u, v)=\lim _{t \rightarrow 0}\left(\frac{u-T_{t}^{D} u}{t}, v\right)
$$

for any non-empty open subset $D$. Finally, for any two non-empty open subsets $D_{1} \subset D_{2}$ of $X$, it is known that

$$
p_{D_{1}}(t, x, y) \leqslant p_{D_{2}}(t, x, y) \leqslant p(t, x, y)
$$

for any $t>0$ and $\mu$-almost all $x, y \in X$, if all of them exist.

Remark 2.1. Let $\left(\left\{X_{t}\right\}_{t \geqslant 0},\left\{\mathbb{P}_{x}\right\}_{x \in X}\right)$ be the Hunt process corresponding to a regular Dirichlet form $(\mathcal{E}, \mathcal{F})$. Let $D_{1} \subset D_{2}$ be two open subsets. Then, for any $t>0$ and $\mu$-almost all $x \in X$,

$$
\begin{aligned}
T_{t}^{D_{1}} u(x) & =\mathbb{E}_{x}^{D_{1}}\left(u\left(X_{t}\right)\right):=\mathbb{E}_{x}\left(\mathbf{1}_{\left\{t<\tau_{D_{1}}\right\}} u\left(X_{t}\right)\right) \\
& \leqslant \mathbb{E}_{x}\left(\mathbf{1}_{\left\{t<\tau_{D_{2}}\right\}} u\left(X_{t}\right)\right)=T_{t}^{D_{2}} u(x) \\
& \leqslant \mathbb{E}_{x}\left(u\left(X_{t}\right)\right)=T_{t} u(x)
\end{aligned}
$$

for any non-negative bounded Borel function $u$, where

$$
\tau_{D}=\inf \left\{t>0: X_{t} \notin D\right\}
$$


is the first exit time of $X_{t}$ from $D$ [12, (4.1.2), p. 135]. Thus, (2.11) easily follows. The analytic proof of (2.11) for the classical case uses the maximum principle (see [7, Lemma 3.3]).

For the form $(\mathcal{E}, \mathcal{F})$, we define the effective resistance $R(x, y)$ for any two points $x, y \in$ $X$ by

$$
R(x, y)^{-1}=\inf \{\mathcal{E}(u): u \in \mathcal{F}, u(x)=1 \text { and } u(y)=0\}
$$

if $x \neq y$, and $R(x, y)=0$ if $x=y$ (possibly $R(x, y)=\infty$ for some points $x, y \in X$ ). By (2.13) we see that

$$
R(x, y)=\sup \left\{\frac{|u(y)-u(x)|^{2}}{\mathcal{E}(u)}: u \in \mathcal{F} \text { and } \mathcal{E}(u)>0\right\}
$$

which gives

$$
|u(y)-u(x)|^{2} \leqslant R(x, y) \mathcal{E}(u), \quad u \in \mathcal{F}, x, y \in X .
$$

We say that $R(x, y)$ satisfies condition (A2) if there exist a number $\gamma>0$ and a constant $c_{2}>0$ such that

$$
c_{2}^{-1} d(y, x)^{\gamma} \leqslant R(x, y) \leqslant c_{2} d(y, x)^{\gamma}
$$

for all $x, y \in X$.

We say that $p(t, x, y)$ satisfies condition (A3) if there exist some constants $a_{i}, b_{i}>0$, $i=1,2$, such that

$$
\begin{aligned}
a_{1} t^{-\alpha / \beta} \exp \left(-b_{1}\left(t^{-1 / \beta} d(x, y)\right)^{\beta_{0}}\right) & \leqslant p(t, x, y) \\
& \leqslant a_{2} t^{-\alpha / \beta} \exp \left(-b_{2}\left(t^{-1 / \beta} d(x, y)\right)^{\beta_{0}}\right)
\end{aligned}
$$

for all $x, y \in X$ and all $0<t \leqslant r_{0}^{\beta}$, where $\beta>0, \beta_{0}=\beta(\beta-1)^{-1}$ and $\alpha$ is the same as in (A1).

We say that the semigroup $\left\{T_{t}\right\}_{t \geqslant 0}$ of the form $(\mathcal{E}, \mathcal{F})$ is of local character if, for any non-empty closed subset $D$ of $X$,

$$
\lim _{t \rightarrow 0} \frac{1}{t}\left(T_{t} \mathbf{1}_{D}\right)(x)=0
$$

uniformly in $x \in X$ satisfying $d(x, D) \geqslant \delta>0$ for any fixed $\delta$ (the function $\mathbf{1}_{D}$ is the indicator of $D$, that is $\mathbf{1}_{D}=1$ on $D$, and $\mathbf{1}_{D}=0$ elsewhere). Clearly, if (2.16) holds, then a regular Dirichlet form $(\mathcal{E}, \mathcal{F})$ is local, by using the dominated convergence theorem. Moreover, if the heat kernel $p(t, x, y)$ of $\left\{T_{t}\right\}$ exists, it follows from (2.16) that $p(t, x, y)$ is of local character as well, that is, for any closed subset $D$ and any fixed $\delta>0$,

$$
\lim _{t \rightarrow 0} \frac{1}{t} \int_{D} p(t, x, y) \mathrm{d} \mu(y)=0
$$

uniformly in $x \in X$ with $d(x, D) \geqslant \delta$. Note that (2.17) was proved in [21] for the diffusion on $X=\mathbb{R}$ by using the probability method (see also $[\mathbf{1 0}]$ ).

We now state the main result of this paper. 
Theorem 2.2. Let $(X, d)$ be a compact metric space satisfying the chain condition. Assume that $(\mathcal{E}, \mathcal{F})$ is an irreducible regular Dirichlet form on $L^{2}(\mu)$. Then the following conditions are equivalent.

(i) The heat kernel $p(t, x, y)$ of $(\mathcal{E}, \mathcal{F})$ exists and satisfies $(A 3)$ with $\alpha<\beta$.

(ii) The measure $\mu$ satisfies (A1) and the effective resistance $R(x, y)$ satisfies (A2) with $\gamma=\beta-\alpha, \beta>1$, and the semigroup $\left\{T_{t}\right\}$ of $(\mathcal{E}, \mathcal{F})$ is of local character.

The proof of Theorem 2.2 will be given in $\S \S 3$ and 5 . It would be interesting to replace the locality of the semigroup $\left\{T_{t}\right\}$ in Theorem 2.2 (ii) by the locality of the form $(\mathcal{E}, \mathcal{F})$. We will explore this for the case of post-critically finite self-similar fractals introduced by Kigami [18].

Remarks 2.3. (1) Condition (A1) implies that $\alpha$ is the Hausdorff dimension of $X[\mathbf{9}]$. The number $\beta$ in (A3) is termed the walk dimension. The spectral dimension $d_{\mathrm{s}}$ of $X$ is determined by the Einstein relation $d_{\mathrm{s}}=2 \alpha / \beta$. We say that $X$ is strongly recurrent if $d_{\mathrm{s}}<2$. Clearly, the $X$ considered in this paper is strongly recurrent.

(2) The upper estimate in (A2) implies the Morrey-Sobolev inequality, that is

$$
|u(y)-u(x)|^{2} \leqslant c d(y, x)^{\gamma} \mathcal{E}(u)
$$

for all $x, y \in X$ and all $u \in \mathcal{F}$, by virtue of (2.15). Thus, $\mathcal{F} \subset C(X)$. For a non-empty proper subset $A$ of $X$, let $\mathcal{F}_{A}$ be equipped with norm $\mathcal{E}(u)^{1 / 2}$. If $\mathcal{F}_{A}$ is not empty, then $\mathcal{F}_{A}$ is compactly embedded in $C(X)$ by using the Ascoli-Arzelà theorem [22, p. 85], since $X$ is compact, that is, any bounded sequence in $\mathcal{F}_{A}$ has a convergent subsequence in $C(X)$. The compact embedding will play an important role; in particular, it implies the existence of Green functions with zero boundary conditions (see $\S 4$ ).

(3) If the upper bound of $R$ in (A2) holds, then $R(x, y)$ is a metric on $X$ (see $\S 4$ ). Thus, the chain condition, together with (A2), implies that $\gamma \leqslant 1$. In fact, let $\left\{x_{k}\right\}_{k=0}^{n}$ be a chain connecting $x, y \in X(x \neq y)$ with $x_{0}=x$ and $x_{n}=y$ for a large integer $n$. We see from (A2) and (2.1) that

$$
\begin{aligned}
c_{2}^{-1} d(y, x)^{\gamma} & \leqslant R(x, y) \leqslant \sum_{k=0}^{n-1} R\left(x_{k}, x_{k+1}\right) \leqslant \sum_{k=0}^{n-1} c_{2} d\left(x_{k}, x_{k+1}\right)^{\gamma} \\
& \leqslant \sum_{k=0}^{n-1} c_{2}\left(c_{0} n^{-1} d(x, y)\right)^{\gamma} \leqslant c n^{1-\gamma} d(x, y)^{\gamma},
\end{aligned}
$$

which implies that $n^{\gamma-1} \leqslant c$. Thus, $\gamma \leqslant 1$, and so $\beta=\alpha+\gamma \leqslant \alpha+1$.

(4) If $R(x, y)$ defined as in (2.13) is shown to be a metric on $X$, we may take $d(y, x)=$ $R(x, y)$ in (A2) with $\gamma=1$. Then Theorem 2.2 says that, for an irreducible regular Dirichlet form $(\mathcal{E}, \mathcal{F})$ on $L^{2}(\mu)$, the conditions (A1) and (2.16) are equivalent to (A3) with $\beta=\alpha+1$ (see [5] for graphs, [17] for post-critically finite fractals with regular harmonic structure and [20] for effective forms). 


\section{Proof of $(\mathrm{A} 3) \Longrightarrow(\mathrm{A} 1)+(\mathrm{A} 2)+(2.16)$}

In this section we show that (i) $\Rightarrow$ (ii) in Theorem 2.2. The fact that (A3) implies (A1) and the upper bound of $R(x, y)$ in (A2) was actually obtained in [16]. We need only to prove the lower bound of $R(x, y)$ in (A2) and (2.16). For the reader's convenience, we outline the whole proof.

Proposition 3.1 (Grigor'yan et al. [16, Theorem 3.2]). Assume that $p(t, x, y)$ satisfies (A3) (without the restriction that $\alpha<\beta$ ). Then $\mu$ satisfies (A1).

Proof. In [16] $X$ is assumed to be unbounded, and so $p(t, x, y)$ satisfies (A3) for all $0<t<\infty$. One can slightly modify the proof in $[\mathbf{1 6}]$ to deal with the case in which $X$ is bounded. Thus, Proposition 3.1 follows.

For $\sigma>0$, we define

$$
W_{\sigma}(u)=\sup _{0<r<1} r^{-2 \sigma} \int_{X}\left\{\frac{1}{\mu(B(x, r))} \int_{B(x, r)}|u(y)-u(x)|^{2} \mathrm{~d} \mu(y)\right\} \mathrm{d} \mu(x)
$$

for $u \in L^{2}(\mu)$.

Proposition 3.2 (Grigor'yan et al. [16, Theorem 4.11 (iii)]). Assume that $\mu$ satisfies (A1). If $\alpha<\beta$, then

$$
|u(y)-u(x)|^{2} \leqslant c d(y, x)^{\beta-\alpha} W_{\beta / 2}(u)
$$

for all $x, y \in X$ and all $u \in C(X)$, for some $c>0$.

Proof. In [16] the embedding (3.2) was obtained for $x, y \in X$ with $d(y, x) \leqslant \frac{1}{3}$. If $d(y, x) \geqslant \frac{1}{3}$, we let $\left\{x_{k}\right\}_{k=0}^{n}$ be a chain connecting $x$ and $y$ such that $d\left(x_{k}, x_{k+1}\right) \leqslant \frac{1}{3}$ for all $0 \leqslant k \leqslant n-1$; this can be done by taking $n=\left[3 c_{0} r_{0}\right]+1$. For $u \in C(X)$, we use (3.2) for each pair $\left(x_{k}, x_{k+1}\right), 0 \leqslant k \leqslant n-1$, and then sum over $k$ to arrive at (3.2) for all $x, y \in X$ with $d(y, x) \geqslant \frac{1}{3}$.

Proposition 3.3 (Grigor'yan et al. [16, Theorem 4.2]). Assume that $p(t, x, y)$ satisfies (A3). Then

$$
c^{-1} W_{\beta / 2}(u) \leqslant \mathcal{E}(u) \leqslant c W_{\beta / 2}(u)
$$

for all $u \in \mathcal{F}$, for some $c>0$.

By (3.2) and (3.3), we see that if $p(t, x, y)$ satisfies (A3) with $\alpha<\beta$, then

$$
|u(y)-u(x)|^{2} \leqslant c d(y, x)^{\beta-\alpha} \mathcal{E}(u)
$$

for all $x, y \in X$ and all $u \in \mathcal{F}$. This immediately gives

$$
R(x, y) \leqslant c d(y, x)^{\beta-\alpha}
$$

for all $x, y \in X$, by virtue of (2.14). So the upper bound of $R(x, y)$ in (A2) follows with $\gamma=\beta-\alpha>0$. It remains to prove the lower bound of $R(x, y)$ in (A2). 
Theorem 3.4. Assume that $p(t, x, y)$ satisfies (A3) with $\alpha<\beta$. Then, for all $x, y \in X$,

$$
R(x, y) \geqslant c^{-1} d(y, x)^{\beta-\alpha} .
$$

Proof. We first show that

$$
\mathcal{E}(p(t, x, \cdot)) \leqslant(e t)^{-1} p(t, x, x)
$$

for all $t>0$ and $x \in X$. Indeed, let $f \in L^{2}(\mu)$, and set

$$
u(t, y)=T_{t} f(y), \quad y \in X .
$$

By the spectral calculus, we have

$$
\mathcal{E}(u(t, \cdot))=\int_{0}^{\infty} \lambda \mathrm{e}^{-2 \lambda t} \mathrm{~d}\left(E_{\lambda} f, f\right),
$$

where $\left\{E_{\lambda}\right\}$ is the spectral representation of the generator of $\left\{T_{t}\right\}$. Noting that

$$
\lambda \mathrm{e}^{-2 \lambda t} \leqslant(2 e t)^{-1}
$$

for $\lambda \geqslant 0$ and $t>0$, we see from above that

$$
\mathcal{E}(u(t, \cdot)) \leqslant(2 e t)^{-1}\|f\|_{2}^{2} .
$$

Fix $x \in X$. Letting $f(y)=p(t, x, y), y \in X$, we obtain $u(t, y)=p(2 t, x, y)$. Thus, we have

$$
\mathcal{E}(p(2 t, x, \cdot)) \leqslant(2 e t)^{-1}\|p(t, x, \cdot)\|_{2}^{2}=(2 e t)^{-1} p(2 t, x, x),
$$

proving (3.7) by replacing $2 t$ by $t$. It follows from (3.7) that, using the upper diagonal bound of $p(t, x, y)$,

$$
\mathcal{E}(p(t, x, \cdot)) \leqslant(e t)^{-1} p(t, x, x) \leqslant a_{2} \mathrm{e}^{-1} t^{-(1+\alpha / \beta)}
$$

for all $0<t \leqslant r_{0}^{\beta}$ and all $x \in X$. Fix $y \in X$. By (A3), (2.15) and (3.8), we have

$$
\begin{aligned}
a_{1} t^{-\alpha / \beta}-a_{2} t^{-\alpha / \beta} \exp \left(-b_{2}\left(t^{-1 / \beta} d(x, y)\right)^{\beta_{0}}\right) & \leqslant p(t, x, x)-p(t, x, y) \\
& \leqslant R(x, y)^{1 / 2} \mathcal{E}(p(t, x, \cdot))^{1 / 2} \\
& \leqslant\left(a_{2} \mathrm{e}^{-1}\right)^{1 / 2} R(x, y)^{1 / 2} t^{-(1+\alpha / \beta) / 2}
\end{aligned}
$$

for all $0<t \leqslant r_{0}^{\beta}$. Let $t=\left(b^{-1} b_{2}\right)^{\beta / \beta_{0}} d(x, y)^{\beta}$, where $b$ is so large that $a_{2} \exp (-b) \leqslant \frac{1}{2} a_{1}$ and $t \leqslant r_{0}^{\beta}$. It follows from above that

$$
\frac{1}{2} a_{1} t^{-\alpha / \beta} \leqslant\left(a_{2} \mathrm{e}^{-1}\right)^{1 / 2} R(x, y)^{1 / 2} t^{-(1+\alpha / \beta) / 2},
$$

and so

$$
R(x, y) \geqslant c t^{(\beta-\alpha) / \beta}=c d(x, y)^{\beta-\alpha},
$$

giving (3.6). 
Finally, we see that (2.17) (or equivalently (2.16)) easily follows from the upper bound of $p(t, x, y)$ in (A3) and the regularity of $\mu$. Indeed, for a closed subset $D$ of $X$ and any point $x \in X$ with $\operatorname{dist}(x, D) \geqslant \delta>0$, we have

$$
\begin{aligned}
t^{-1} \int_{D} p(t, x, y) \mathrm{d} \mu(y) & \leqslant t^{-1} \int_{B(x, \delta)^{\mathrm{c}}} p(t, x, y) \mathrm{d} \mu(y) \\
& \leqslant c t^{-1} \int_{\frac{1}{2} \delta t^{-1 / \beta}}^{\infty} s^{\alpha-1} \exp \left(-c^{\prime} s^{\beta_{0}}\right) \mathrm{d} s \rightarrow 0
\end{aligned}
$$

as $t \rightarrow 0$ (see, for example, $[\mathbf{1 6},(3.7)])$. If (A3) holds and $(X, d)$ satisfies the chain condition, then $\beta \geqslant 2$ (see [16, (4.27), p. 2081]).

\section{Green functions}

In order to prove the other direction in Theorem 2.2, we need to investigate the existence of Green functions with zero boundary conditions. In this section, we assume only that $(\mathcal{E}, \mathcal{F})$ is an irreducible regular Dirichlet form, and that the upper bound of $R$ in (A2) holds. The variational problem (2.13) or (4.1) below possesses a unique solution, leading to the existence of Green functions with boundary conditions. This also generalizes the results on Green functions with boundary having finite points [19] to the case where the boundary may have infinite points.

For any non-empty subset $A$ of $X$, we define the effective resistance $R(x, A)$ between any point $x \in X$ and $A$ by $R(x, A)=0$ if $x \in A$, and

$$
R(x, A)^{-1}=\inf \left\{\mathcal{E}(u): u \in \mathcal{F}_{A^{c}}^{x}\right\}
$$

if $x \notin A$, where

$$
\mathcal{F}_{A^{\mathrm{c}}}^{x}:=\left\{u \in \mathcal{F}: u(x)=1 \text { and }\left.u\right|_{A}=0\right\}, \quad x \notin A .
$$

(Recall that $\mathcal{F} \subset C(X)$ by Remark $2.3(2)$, and so $u \in \mathcal{F}$ is defined pointwise on $X$.) Note that $\mathcal{F}_{A^{\mathrm{c}}}^{x}$ may be empty for some subsets $A$ of $X$. However, we have the following.

Proposition 4.1. Assume that $(\mathcal{E}, \mathcal{F})$ is an irreducible regular Dirichlet form on $L^{2}(\mu)$. Then, for any two disjoint non-empty closed subsets $A$ and $B$ of $X$,

$$
\left\{u \in \mathcal{F}:\left.u\right|_{A}=0,\left.u\right|_{B}=1\right\} \neq \emptyset
$$

In particular, the set $\mathcal{F}_{A^{\mathrm{c}}}^{x}$ is not empty for any $x \notin A$ and any non-empty closed subset $A \subset X$.

Proof. Let $A$ and $B$ be two closed subsets of $X$ with $A \cap B=\emptyset$. By the Urysohn theorem [22, p. 7], there exists a real-valued continuous function $v$ on $X$ such that $0 \leqslant v \leqslant 1$ on $X$, and $\left.v\right|_{A}=0$ and $\left.v\right|_{B}=1$. Since $\mathcal{E}$ is regular, there is a function $u_{1} \in \mathcal{F}$ such that $\left.u_{1}\right|_{A} \leqslant \frac{1}{3}$ and $\left.u_{1}\right|_{B} \geqslant \frac{2}{3}$. Since $\mathcal{E}$ is irreducible, we see that $u_{1}-\frac{1}{3} \in \mathcal{F}$. Define $u_{+}=0 \vee u$. Let

$$
u=\left(3\left(u_{1}-\frac{1}{3}\right)_{+}\right) \wedge 1 .
$$

It is easily seen that $u$ is the desired function by using the Markov property of $\mathcal{E}$. 
Proposition 4.2. Assume that $(\mathcal{E}, \mathcal{F})$ is an irreducible regular Dirichlet form on $L^{2}(\mu)$, and that the upper bound of $R$ in (A2) holds. Then, for any non-empty closed subset $A$ of $X$ and any $x_{0} \notin A$, the variational problem

$$
\lambda_{0}=\inf \left\{\mathcal{E}(u): u \in \mathcal{F}_{A^{\mathrm{c}}}^{x_{0}}\right\}
$$

possesses a unique solution in $[0,1]$, that is, there is a unique function $\psi_{A}^{x_{0}} \in \mathcal{F}_{A^{c}}^{x_{0}}$ with $0 \leqslant \psi_{A}^{x_{0}} \leqslant 1$ on $X$ such that $\lambda_{0}=\mathcal{E}\left(\psi_{A}^{x_{0}}\right)$.

Proof. The proof is standard, by using the compact embedding theorem. We outline the proof for the reader's convenience. Since $\mathcal{F}_{A^{\mathrm{c}}}^{x_{0}}$ is not empty, we see that $\lambda_{0}<\infty$. Let $\left\{u_{n}\right\}_{n \geqslant 1}$ be a minimizing sequence for (4.2), that is $\left\{u_{n}\right\}_{n \geqslant 1} \in \mathcal{F}_{A^{\mathrm{c}}}^{x_{0}}$ and $\mathcal{E}\left(u_{n}\right) \rightarrow$ $\lambda_{0}$ as $n \rightarrow \infty$. The compact embedding (see Remark 2.3(2)) implies that there is a subsequence of $\left\{u_{n}\right\}$ (still denoted by $\left\{u_{n}\right\}$ ) and a function $\psi_{A}^{x_{0}} \in C(X)$ such that $\left\{u_{n}\right\}$ uniformly converges to $\psi_{A}^{x_{0}}$ as $n \rightarrow \infty$. Clearly, $\psi_{A}^{x_{0}}\left(x_{0}\right)=1$ and $\left.\psi_{A}^{x_{0}}\right|_{A}=0$. We show that $\psi_{A}^{x_{0}} \in \mathcal{F}$. In fact, since $\mathcal{E}_{t}(u)$ increases to $\mathcal{E}(u)$ as $t \rightarrow 0$, from $(2.9)$, using the dominated convergence theorem, we have

$$
\mathcal{E}_{t}\left(\psi_{A}^{x_{0}}\right)=\lim _{n \rightarrow \infty} \mathcal{E}_{t}\left(u_{n}\right) \leqslant \lim _{n \rightarrow \infty} \mathcal{E}\left(u_{n}\right)=\lambda_{0}
$$

for $t>0$, which gives that $\mathcal{E}\left(\psi_{A}^{x_{0}}\right) \leqslant \lambda_{0}<\infty$. Thus, $\psi_{A}^{x_{0}} \in \mathcal{F}$. Clearly, $\mathcal{E}\left(\psi_{A}^{x_{0}}\right)=\lambda_{0}$. To show that $\psi_{A}^{x_{0}}$ is unique, let $v \in \mathcal{F}_{A^{\mathrm{c}}}^{x_{0}}$, satisfying $\mathcal{E}(v)=\lambda_{0}$. Note that

$$
0 \leqslant \mathcal{E}\left(\frac{v-\psi_{A}^{x_{0}}}{2}\right)=\frac{1}{2}\left(\mathcal{E}(v)+\mathcal{E}\left(\psi_{A}^{x_{0}}\right)\right)-\mathcal{E}\left(\frac{v+\psi_{A}^{x_{0}}}{2}\right) \leqslant 0
$$

since $\mathcal{E}\left(\frac{1}{2}\left(v+\psi_{A}^{x_{0}}\right)\right) \geqslant \lambda_{0}$, and so $v-\psi_{A}^{x_{0}}$ is constant by virtue of the irreducibility of $\mathcal{E}$. Thus, $v=\psi_{A}^{x_{0}}$ on $X$. It remains to show that $0 \leqslant \psi_{A}^{x_{0}} \leqslant 1$. But this easily follows from the Markov property of $\mathcal{E}$ and the uniqueness. Indeed, let $u=\left(0 \vee \psi_{A}^{x_{0}}\right) \wedge 1$. By the Markov property of $\mathcal{E}$, we see that $u \in \mathcal{F}_{A^{\mathrm{c}}}^{x_{0}}$ and $\mathcal{E}(u) \leqslant \mathcal{E}\left(\psi_{A}^{x_{0}}\right)$. The uniqueness proved above implies that $\psi_{A}^{x_{0}}=u \in[0,1]$.

For any two distinct points $x_{0}, y_{0} \in X$, letting $A=\left\{y_{0}\right\}$, we see from Proposition 4.2 that there is a unique function $\psi_{y_{0}}^{x_{0}}$ with the property that $\psi_{y_{0}}^{x_{0}}\left(x_{0}\right)=1$ and $\psi_{y_{0}}^{x_{0}}\left(y_{0}\right)=0$ such that

$$
R\left(x_{0}, y_{0}\right)^{-1}=\mathcal{E}\left(\psi_{y_{0}}^{x_{0}}\right)
$$

Next we claim that, for any closed set $A \subset X$ and $x_{0} \in X$, the function $\psi_{A}^{x_{0}} \in \mathcal{F}_{A^{\mathrm{c}}}^{x_{0}}$ is the solution to (4.1) with $x=x_{0}$ if and only if

$$
\mathcal{E}\left(\psi_{A}^{x_{0}}, v\right)=0
$$

for all $v \in \mathcal{F}$ satisfying $\left.v\right|_{A \cup\left\{x_{0}\right\}}=0$. In fact, if $\psi_{A}^{x_{0}}$ is the solution to (4.1), we find that $\psi_{A}^{x_{0}}+t v \in \mathcal{F}_{A^{c}}^{x_{0}}$ for any $t \in \mathbb{R}$ and $v \in \mathcal{F}$ satisfying $\left.v\right|_{A \cup\left\{x_{0}\right\}}=0$. Thus,

$$
\mathcal{E}\left(\psi_{A}^{x_{0}}\right) \leqslant \mathcal{E}\left(\psi_{A}^{x_{0}}+t v\right)=\mathcal{E}\left(\psi_{A}^{x_{0}}\right)+2 t \mathcal{E}\left(\psi_{A}^{x_{0}}, v\right)+t^{2} \mathcal{E}(v)
$$


which means that $2 t \mathcal{E}\left(\psi_{A}^{x_{0}}, v\right)+t^{2} \mathcal{E}(v) \geqslant 0$ for any $t \in \mathbb{R}$. Therefore, (4.4) follows. Conversely, if there is a function $\psi_{A}^{x_{0}} \in \mathcal{F}_{A^{\mathrm{c}}}^{x_{0}}$ such that (4.4) holds, then we let $v=f-\psi_{A}^{x_{0}}$ for any $f \in \mathcal{F}_{A^{\mathrm{c}}}^{x_{0}}$, and obtain

$$
\mathcal{E}(f)=\mathcal{E}\left(v+\psi_{A}^{x_{0}}\right)=\mathcal{E}(v)+2 \mathcal{E}\left(\psi_{A}^{x_{0}}, v\right)+\mathcal{E}\left(\psi_{A}^{x_{0}}\right)=\mathcal{E}(v)+\mathcal{E}\left(\psi_{A}^{x_{0}}\right) \geqslant \mathcal{E}\left(\psi_{A}^{x_{0}}\right)
$$

Thus, $\psi_{A}^{x_{0}}$ is the solution to (4.1), proving the claim.

For any non-empty closed set $A \subset X$ and any continuous function $\varphi$ defined on $A$, we say that a function $f \in \mathcal{F}$ is harmonic on $A^{\mathrm{c}}$ with the boundary condition $\left.f\right|_{A}=\varphi$ if

$$
\mathcal{E}(f, v)=0
$$

for any $v \in \mathcal{F}_{A^{\mathrm{c}}}$. Thus, by (4.4), the function $\psi_{A}^{x_{0}}$ is harmonic on $X \backslash A \cup\left\{x_{0}\right\}$ with boundary condition on $\left.\psi_{A}^{x_{0}}\right|_{A}=0$ and $\psi_{A}^{x_{0}}\left(x_{0}\right)=1$. Note that a harmonic function is uniquely determined by its boundary condition, using the irreducibility of $\mathcal{E}$.

Definition 4.3. Let $A$ be a closed subset of $X$ and $x_{0} \in X$. Define the Green function $g_{A}^{x_{0}}(\cdot)=R\left(x_{0}, A\right) \psi_{A}^{x_{0}}(\cdot)$ if $x_{0} \notin A$, and $g_{A}^{x_{0}} \equiv 0$ if $x_{0} \in A$.

Remark 4.4. The Green function defined above is the same as that introduced in [19] if $A$ is a finite subset of $X$. If $A=\left\{y_{0}\right\}$ for $y_{0} \in X$, we write the Green function $g_{y_{0}}^{x_{0}}(\cdot)=R\left(x_{0}, y_{0}\right) \psi_{y_{0}}^{x_{0}}(\cdot)$.

The Green function $g_{A}^{x_{0}}$ has the following properties (see [19] if $A$ is a finite set).

(i) $g_{A}^{x_{0}} \geqslant 0$ on $X$, and $g_{A}^{x_{0}}(x)=0$ if $x \in A$ or $x_{0} \in A$.

(ii) $g_{A}^{x}(y)=g_{A}^{y}(x)$ for $x, y \in X$ (see (4.7), below).

(iii) $\mathcal{E}\left(g_{A}^{x_{0}}\right)=R\left(x_{0}, A\right)=g_{A}^{x_{0}}\left(x_{0}\right)$.

(iv) $g_{A}^{x_{0}}\left(x_{0}\right) \geqslant g_{A}^{x_{0}}(x)$ for all $x \in X\left(\right.$ since $0 \leqslant \psi_{y_{0}}^{x_{0}} \leqslant 1$ on $\left.X\right)$.

Lemma 4.5. Let $g_{A}^{x_{0}}$ be the Green function defined above for any closed subset $A$ of $X$ and any point $x_{0} \in X$. Then, for any $u \in \mathcal{F}_{A^{\mathrm{c}}}$,

$$
\mathcal{E}\left(g_{A}^{x_{0}}, u\right)=u\left(x_{0}\right)
$$

Remark 4.6. It is easy to see that (4.5) fails if $u \notin \mathcal{F}_{A^{\mathrm{c}}}$, for example, by letting $u \equiv 1$ on $X$.

Proof. If $x_{0} \in A$, nothing can be proved. Now let $x_{0} \notin A$. It suffices to show that

$$
\mathcal{E}\left(\psi_{A}^{x_{0}}, u\right)=u\left(x_{0}\right) R\left(x_{0}, A\right)^{-1}
$$

for $u \in \mathcal{F}_{A^{\mathrm{c}}}$. We assume that $u\left(x_{0}\right) \neq 0$; otherwise, (4.5) follows from (4.4). Let $v(x)=$ $\psi_{A}^{x_{0}}(x)-u\left(x_{0}\right)^{-1} u(x)$ for $x \in X$. Clearly, $v\left(x_{0}\right)=0$ and $\left.v\right|_{A}=0$. Thus, it follows from (4.4) that

$$
0=\mathcal{E}\left(\psi_{A}^{x_{0}}, v\right)=\mathcal{E}\left(\psi_{A}^{x_{0}}\right)-u\left(x_{0}\right)^{-1} \mathcal{E}\left(\psi_{A}^{x_{0}}, u\right)
$$

giving (4.6) by using the fact that $\mathcal{E}\left(\psi_{A}^{x_{0}}\right)=R\left(x_{0}, A\right)^{-1}$. 
Since $g_{A}^{y_{0}} \in \mathcal{F}_{A^{c}}$ for any $y_{0} \in X$, it follows from (4.5) that

$$
\mathcal{E}\left(g_{A}^{x_{0}}, g_{A}^{y_{0}}\right)=g_{A}^{x_{0}}\left(y_{0}\right)=g_{A}^{y_{0}}\left(x_{0}\right)
$$

for any $x_{0}, y_{0} \in X$. If $A=\left\{y_{0}\right\}$, from (4.5) we have

$$
\mathcal{E}\left(g_{y_{0}}^{x_{0}}, u\right)=u\left(x_{0}\right)
$$

for any $u \in \mathcal{F}$ with $u\left(y_{0}\right)=0$. For general $u \in \mathcal{F}$, we let $\bar{u}=u-u\left(y_{0}\right)$, and (4.8) applied to $\bar{u}$ gives

$$
\mathcal{E}\left(g_{y_{0}}^{x_{0}}, u\right)=u\left(x_{0}\right)-u\left(y_{0}\right)
$$

for any $u \in \mathcal{F}$ and any $x_{0}, y_{0} \in X$.

Lemma 4.7. Let $A$ be a non-empty closed subset of $X$ and $x_{0} \in X$. Then

$$
R\left(x_{0}, A\right) \leqslant R\left(x_{0}, y_{0}\right)+R\left(y_{0}, A\right)
$$

for any $x_{0}, y_{0} \in X$. In particular, if $A=\left\{z_{0}\right\}$, then $R$ satisfies the triangle inequality

$$
R\left(x_{0}, z_{0}\right) \leqslant R\left(x_{0}, y_{0}\right)+R\left(y_{0}, z_{0}\right)
$$

for any $x_{0}, y_{0}, z_{0} \in X$.

Proof. Let $x_{0}, y_{0} \in X, x_{0} \neq y_{0}$. Motivated by [19], we let $h(x)=g_{A}^{x_{0}}(x)-g_{A}^{y_{0}}(x)$ for $x \in X$. Note that $h\left(x_{0}\right) \geqslant 0$ and $h\left(y_{0}\right) \leqslant 0$, since $g_{A}^{x_{0}}\left(x_{0}\right) \geqslant g_{A}^{x_{0}}(x)$ for any $x \in X$. Since $h \in \mathcal{F}_{A^{\mathrm{c}}}$, we see from (4.5) that

$$
\mathcal{E}(h)=\mathcal{E}\left(g_{A}^{x_{0}}-g_{A}^{y_{0}}, h\right)=h\left(x_{0}\right)-h\left(y_{0}\right),
$$

which combines with (2.15) to give

$$
\left(h\left(x_{0}\right)-h\left(y_{0}\right)\right)^{2} \leqslant R\left(x_{0}, y_{0}\right) \mathcal{E}(h)=R\left(x_{0}, y_{0}\right)\left(h\left(x_{0}\right)-h\left(y_{0}\right)\right) .
$$

Therefore,

$$
0 \leqslant h\left(x_{0}\right) \leqslant h\left(x_{0}\right)-h\left(y_{0}\right) \leqslant R\left(x_{0}, y_{0}\right)
$$

and so

$$
0 \leqslant g_{A}^{x_{0}}\left(x_{0}\right)-g_{A}^{y_{0}}\left(x_{0}\right)=h\left(x_{0}\right) \leqslant R\left(x_{0}, y_{0}\right) .
$$

Hence,

$$
R\left(x_{0}, A\right)=g_{A}^{x_{0}}\left(x_{0}\right) \leqslant R\left(x_{0}, y_{0}\right)+g_{A}^{y_{0}}\left(x_{0}\right) \leqslant R\left(x_{0}, y_{0}\right)+R\left(y_{0}, A\right),
$$

proving (4.10).

Remark 4.8. The result in (4.10) was obtained by Kigami $[\mathbf{1 9}]$ for the case when $A$ is a finite subset of $X$. Note that (4.11) implies that $R$ is a metric on $X$ (we assume that the upper bound in (A2) holds, so $R(x, y)<\infty$ for any $x, y \in X$; this is because, if $R\left(x_{0}, y_{0}\right)=\infty$ for some $x_{0}, y_{0} \in X$, then $\mathcal{E}\left(\psi_{y_{0}}^{x_{0}}\right)=R\left(x_{0}, y_{0}\right)^{-1}=0$, which would imply that $\psi_{y_{0}}^{x_{0}} \equiv$ const., which is a contradiction by Proposition 4.2). We call $R$ the effective resistance metric on $X$. 
For $y_{0} \in A$, we observe that $R\left(y_{0}, A\right)=0$, and (4.10) implies that

$$
R\left(x_{0}, A\right) \leqslant \inf _{y \in A} R\left(x_{0}, y\right)
$$

for any $x_{0} \in X$ and any closed subset $A$ of $X$. We next state that the Green function $g_{A}^{x_{0}}$ is uniformly Lipschitz in terms of $R$ (see [19] for a finite subset $A$ of $X$ ).

Lemma 4.9. Let $A$ be a closed subset of $X$ and $x_{0} \in X$. Then

$$
\left|g_{A}^{x_{0}}(x)-g_{A}^{x_{0}}(y)\right| \leqslant R(x, y)
$$

for any $x, y \in X$.

Proof. The proof given here is motivated by [19, Lemma 4.9, p. 413]. Fix $x_{0}, x \in X$ temporally. Assume that $x_{0}, x \notin A$; otherwise (4.14) is clear. By (4.4), the function

$$
u(y)=g_{A}^{x}(x) g_{A}^{x_{0}}(y)-g_{A}^{x_{0}}(x) g_{A}^{x}(y), \quad y \in X
$$

is harmonic on $X \backslash A \cup\left\{x_{0}, x\right\}$ with boundary conditions on $A \cup\left\{x_{0}, x\right\}$. Note that $u=0$ on $A$, and $u(x)=0$ and

$$
u\left(x_{0}\right)=g_{A}^{x}(x) g_{A}^{x_{0}}\left(x_{0}\right)-g_{A}^{x_{0}}(x) g_{A}^{x}\left(x_{0}\right) \geqslant 0 .
$$

Therefore, we have $u \geqslant 0$ on $X$. $^{*}$ Thus,

$$
g_{A}^{x}(x) g_{A}^{x_{0}}(y) \geqslant g_{A}^{x_{0}}(x) g_{A}^{x}(y)
$$

for all $x_{0}, x, y \in X$. Therefore,

$$
\begin{aligned}
g_{A}^{x_{0}}(x)-g_{A}^{x_{0}}(y) & \leqslant g_{A}^{x_{0}}(x)-g_{A}^{x}(x)^{-1} g_{A}^{x_{0}}(x) g_{A}^{x}(y) \\
& =g_{A}^{x_{0}}(x) g_{A}^{x}(x)^{-1}\left(g_{A}^{x}(x)-g_{A}^{x}(y)\right) \\
& \leqslant g_{A}^{x}(x)-g_{A}^{x}(y) \\
& \leqslant R(x, y)
\end{aligned}
$$

for all $x_{0}, x, y \in X$, where the last inequality follows from (4.12). Exchanging $x$ and $y$ yields

$$
g_{A}^{x_{0}}(y)-g_{A}^{x_{0}}(x) \leqslant R(y, x)
$$

whence (4.14) follows.

* In fact, if $u\left(x_{0}\right)=0$, then $u \equiv 0$ on $X$ by uniqueness, and if $u\left(x_{0}\right)>0$, then the function $u / u\left(x_{0}\right)$ satisfies the variational problem

$$
\inf \left\{\mathcal{E}(u):\left.u\right|_{A \cup\{x\}}=0 \text { and } u\left(x_{0}\right)=1\right\},
$$

and a similar argument to Proposition 4.2 in which $A$ is replaced by $A \cup\{x\}$ shows that $0 \leqslant u / u\left(x_{0}\right) \leqslant 1$ on $X$. 


\section{Proof of $(\mathrm{A} 1)+(\mathrm{A} 2)+(2.16) \Longrightarrow(\mathrm{A} 3)$}

In this section we prove the other direction in Theorem 2.2, that is (A1), (A2) and (2.16) will imply (A3). We first obtain off-diagonal upper bounds of $p(t, x, y)$. The key is to estimate the solution of a linear elliptic equation (5.40) in the ball (see (5.48), below). The Green functions discussed above will be used. The locality of the semigroup plays an important role, which leads to a local maximum principle. We then derive lower bounds of $p(t, x, y)$ in a standard way by using the upper bound of $p(t, x, y),(2.15)$ and the chain condition.

\subsection{On-diagonal upper bounds}

Theorem 5.1. Let $(X, d, \mu)$ be a measure metric space and let $(\mathcal{E}, \mathcal{F})$ be a Dirichlet form (not necessarily local). If $\mu$ satisfies the lower bound in (A1) and $R$ satisfies the upper bound in $(A 2)$, then the heat kernel $p(t, x, y)$ of $(\mathcal{E}, \mathcal{F})$ exists, is continuous on $X \times X$ for each $t>0$ and satisfies

$$
p(t, x, y) \leqslant c t^{-\alpha / \beta}
$$

for all $x, y \in X$ and $0<t \leqslant r_{0}^{\beta}$, where $\beta=\gamma+\alpha$.

Proof. Let $f$ be a non-negative bounded function on $X$ with $\|f\|_{1} \leqslant 1$. For $0<t \leqslant r_{0}^{\beta}$, we show that there exists a constant $c$ independent of $f$ and $t$ such that

$$
\left\|T_{t} f\right\|_{\infty} \leqslant c t^{-\alpha / \beta} .
$$

The proof given here is motivated by [5] for graphs, but we do not assume a priori the existence of the heat kernel. To see this, note that $\left\|T_{t} f\right\|_{1} \leqslant\|f\|_{1} \leqslant 1$, and

$$
\left\|T_{t / 2} f\right\|_{2}^{2}=\left(T_{t / 2} f, T_{t / 2} f\right)=\left(f, T_{t} f\right) \leqslant\left\|T_{t} f\right\|_{\infty}\|f\|_{1} \leqslant\left\|T_{t} f\right\|_{\infty}, \quad t>0 .
$$

Fix $x_{0} \in X$, and define $B_{0}:=B\left(x_{0}, t^{1 / \beta}\right)$. Since

$$
\int_{B_{0}} T_{t} f(x) \mathrm{d} \mu(x) \leqslant\left\|T_{t} f\right\|_{1} \leqslant 1
$$

using $(a+b)^{2} \leqslant 2\left(a^{2}+b^{2}\right)$ and Hölder's inequality, we have

$$
\begin{aligned}
T_{t} f\left(x_{0}\right)^{2} & =\left(\mu\left(B_{0}\right)^{-1} \int_{B_{0}}\left(\left(T_{t} f\left(x_{0}\right)-T_{t} f(x)\right)+T_{t} f(x)\right) \mathrm{d} \mu(x)\right)^{2} \\
& \leqslant 2\left(\mu\left(B_{0}\right)^{-1} \int_{B_{0}}\left(T_{t} f\left(x_{0}\right)-T_{t} f(x)\right) \mathrm{d} \mu(x)\right)^{2}+2\left(\mu\left(B_{0}\right)^{-1} \int_{B_{0}} T_{t} f(x) \mathrm{d} \mu(x)\right)^{2} \\
& \leqslant 2 \mu\left(B_{0}\right)^{-1} \int_{B_{0}}\left(T_{t} f\left(x_{0}\right)-T_{t} f(x)\right)^{2} \mathrm{~d} \mu(x)+2 \mu\left(B_{0}\right)^{-2}
\end{aligned}
$$

Observing that

$$
\mathcal{E}\left(T_{t} f\right)=-\frac{1}{2} \frac{\partial}{\partial t}\left\|T_{t} f\right\|_{2}^{2}, \quad t>0
$$


it follows that, using (2.15) and the upper bound of $R$ in (A2),

$$
\begin{aligned}
\left(T_{t} f\left(x_{0}\right)-T_{t} f(x)\right)^{2} & \leqslant R\left(x_{0}, x\right) \mathcal{E}\left(T_{t} f\right) \leqslant c_{2} d\left(x_{0}, x\right)^{\beta-\alpha} \mathcal{E}\left(T_{t} f\right) \\
& \leqslant-\frac{c_{2}}{2} t^{1-\alpha / \beta} \frac{\partial}{\partial t}\left\|T_{t} f\right\|_{2}^{2}
\end{aligned}
$$

for $x \in B_{0}$ and $t>0$. Therefore,

$$
2 \mu\left(B_{0}\right)^{-1} \int_{B_{0}}\left(T_{t} f\left(x_{0}\right)-T_{t} f(x)\right)^{2} \mathrm{~d} \mu(x) \leqslant-c_{2} t^{1-\alpha / \beta} \frac{\partial}{\partial t}\left\|T_{t} f\right\|_{2}^{2},
$$

which combines with (5.4) to yield that, using the lower bound of $\mu$ in (A1),

$$
T_{t} f\left(x_{0}\right)^{2} \leqslant-c_{2} t^{1-\alpha / \beta} \frac{\partial}{\partial t}\left\|T_{t} f\right\|_{2}^{2}+c t^{-2 \alpha / \beta}
$$

for $0<t \leqslant r_{0}^{\beta}$, where $c$ is independent of $x_{0}, t$ and $f$. Set $\phi(t)=\left\|T_{t} f\right\|_{\infty}$. Note that $\phi$ is decreasing on $(0, \infty)$ because, for $s<t$,

$$
\begin{aligned}
\phi(t) & =\left\|T_{t} f\right\|_{\infty}=\sup _{\|g\|_{1}=1}\left(T_{t} f, g\right)=\sup _{\|g\|_{1}=1}\left(T_{t-s}\left(T_{s} f\right), g\right) \\
& =\sup _{\|g\|_{1}=1}\left(T_{s} f, T_{t-s} g\right) \leqslant\left\|T_{s} f\right\|_{\infty} \sup _{\|g\|_{1}=1}\left\|T_{t-s} g\right\|_{1} \leqslant\left\|T_{s} f\right\|_{\infty}=\phi(s) .
\end{aligned}
$$

Since $x_{0}$ is an arbitrary point in $X$, we see from (5.5) that

$$
\frac{\partial}{\partial t}\left\|T_{t} f\right\|_{2}^{2} \leqslant c t^{-1-\alpha / \beta}-c^{\prime} t^{-1+\alpha / \beta} \phi(t)^{2} .
$$

Integrating (5.6) over $\left(\frac{1}{2} t, t\right)$, and then using (5.3) and the monotonicity of $\phi$, we obtain

$$
\begin{aligned}
-\phi(t) & \leqslant\left\|T_{t} f\right\|_{2}^{2}-\left\|T_{t / 2} f\right\|_{2}^{2} \\
& \leqslant c \int_{t / 2}^{t} s^{-1-\alpha / \beta} \mathrm{d} s-c^{\prime} \int_{t / 2}^{t} s^{-1+\alpha / \beta} \phi(s)^{2} \mathrm{~d} s \\
& \leqslant c t^{-\alpha / \beta}-c^{\prime} \phi(t)^{2} \int_{t / 2}^{t} s^{-1+\alpha / \beta} \mathrm{d} s \\
& =c t^{-\alpha / \beta}-c^{\prime} t^{\alpha / \beta} \phi(t)^{2} .
\end{aligned}
$$

Therefore,

$$
c^{\prime} t^{\alpha / \beta} \phi(t)^{2}-\phi(t)-c t^{-\alpha / \beta} \leqslant 0
$$

which gives

$$
0 \leqslant \phi(t) \leqslant \frac{1+\sqrt{1+4 c c^{\prime}}}{2 c^{\prime} t^{\alpha / \beta}}=c t^{-\alpha / \beta},
$$

proving (5.2) for $0<t \leqslant r_{0}^{\beta}$. The estimate (5.2) implies that the operator $T_{t}$ is ultracontractive from $L^{1}(\mu)$ to $L^{\infty}(\mu)$ for $0<t \leqslant r_{0}^{\beta}$. Thus, the heat kernel $p(t, x, y)$ of $(\mathcal{E}, \mathcal{F})$ exists [13], and (5.1) holds for all $0<t \leqslant r_{0}^{\beta}$ and almost all $x, y \in X$. Finally, using (3.7) and (5.1), we obtain the (Hölder) continuity of $p(t, x, y)$ by noting that

$$
\left|p\left(t, x, y_{1}\right)-p\left(t, x, y_{2}\right)\right|^{2} \leqslant R\left(y_{1}, y_{2}\right) \mathcal{E}(p(t, x, \cdot)) \leqslant c t^{-(1+\alpha / \beta)} d\left(y_{1}, y_{2}\right)^{\beta-\alpha} .
$$

This completes the proof. 
The following proposition shows that the semigroup $\left\{T_{t}\right\}$ of the Dirichlet form $(\mathcal{E}, \mathcal{F})$ is a Feller semigroup if the hypotheses in Theorem 5.1 hold.

Proposition 5.2. Assume that the hypotheses in Theorem 5.1 hold. Then $T_{t} u$ is continuous on $X$ for any bounded function $u$ on $X$ and any $t>0$. Moreover, $\left\{T_{t}\right\}$ is strongly continuous with uniform norm, that is

$$
\lim _{t \rightarrow 0}\left\|T_{t} u-u\right\|_{C(X)}=0, \quad u \in C(X) .
$$

Proof. Let $u$ be bounded on $X$. For a point $x_{0} \in X$, let $\left\{x_{k}\right\}_{k \geqslant 1}$ be a sequence of points in $X$ such that $x_{k} \rightarrow x_{0}$ as $k \rightarrow \infty$. Since the heat kernel $p(t, x, y)$ is continuous in $x$ for $t>0$ and $y \in X$, and $p\left(t, x_{k}, y\right) \leqslant c t^{-\alpha / \beta}$ by (5.1), using the dominated convergence theorem, we have

$$
\lim _{k \rightarrow \infty} T_{t} u\left(x_{k}\right)=\lim _{k \rightarrow \infty} \int_{X} p\left(t, x_{k}, y\right) u(y) \mathrm{d} \mu(y)=\int_{X} p\left(t, x_{0}, y\right) u(y) \mathrm{d} \mu(y)=T_{t} u\left(x_{0}\right) .
$$

Thus, $T_{t} u$ is continuous on $X$ for each $t>0$. It remains to prove (5.8). To this end, note that, for any $u \in \mathcal{F}$,

$$
\mathcal{E}\left(T_{t} u-u\right)=\int_{0}^{\infty} \lambda\left(\mathrm{e}^{-\lambda t}-1\right)^{2} \mathrm{~d}\left(E_{\lambda} u, u\right)
$$

by using the spectral calculus, where $\left\{E_{\lambda}\right\}$ is the spectral family associated with the generator $H$ of the form $(\mathcal{E}, \mathcal{F})$. Thus, by the dominated convergence theorem,

$$
\lim _{t \rightarrow 0} \mathcal{E}\left(T_{t} u-u\right)=\lim _{t \rightarrow 0} \int_{0}^{\infty} \lambda\left(\mathrm{e}^{-\lambda t}-1\right)^{2} \mathrm{~d}\left(E_{\lambda} u, u\right)=0 .
$$

On the other hand, from the upper bound in (A2) and (2.15) we have

$$
\|u\|_{C(X)}^{2} \leqslant c\left(\mathcal{E}(u)+\|u\|_{2}^{2}\right)
$$

for all $u \in \mathcal{F}$. Replacing $u$ by $T_{t} u-u$ in (5.11), we see that

$$
\lim _{t \rightarrow 0}\left\|T_{t} u-u\right\|_{C(X)}^{2} \leqslant c \lim _{t \rightarrow 0}\left(\mathcal{E}\left(T_{t} u-u\right)+\left\|T_{t} u-u\right\|_{2}^{2}\right)=0
$$

for any $u \in \mathcal{F}$, by virtue of $(5.10)$ and $(2.4)$. Since $(\mathcal{E}, \mathcal{F})$ is regular and $\left\|T_{t} u\right\|_{C(X)} \leqslant$ $\|u\|_{C(X)}$, we can easily see that (5.12) also holds for $u \in C(X)$.

As in Theorem 5.1 and Proposition 5.2, we observe that, for any open subset $D$, the heat kernel $p_{D}(t, x, y)$ of the form $\left(\mathcal{E}, \mathcal{F}_{D}\right)$ also exists and is jointly continuous, and

$$
\lim _{t \rightarrow 0}\left\|T_{t}^{D} u-u\right\|_{C(D)}=0
$$

for any $u \in C_{0}(D)$.

We remark here that (5.8) was proved in $[\mathbf{1 8}$, Lemma 5.2.7, p. 167] if $X$ is a postcritically finite fractal with regular harmonic structure. 


\subsection{Off-diagonal upper bounds}

We first give estimates of the effective resistance between any point $x_{0} \in X$ and $B\left(x_{0}, r\right)^{\mathrm{c}}$ with $r \in\left(0, r_{0}\right]$. The locality of the form $(\mathcal{E}, \mathcal{F})$ will be used.

Proposition 5.3. Let $(X, d, \mu)$ be a measure metric space satisfying the chain condition. Assume that $(\mathcal{E}, \mathcal{F})$ is a local regular Dirichlet form. If $R$ satisfies $(A 2)$, then, for all $x_{0} \in X$ and $0<r \leqslant r_{0}$,

$$
R\left(x_{0}, B\left(x_{0}, r\right)^{\mathrm{c}}\right) \geqslant c r^{\gamma}
$$

Proof. The proof is essentially the same as in [5] for graphs or in [20] for effective forms. Let $D=\bar{B}\left(x_{0}, r\right) \backslash B\left(x_{0}, \frac{1}{2} r\right)$. For $y \in D$, let $\psi_{y}^{x_{0}}$ be the unique function such that $R\left(x_{0}, y\right)^{-1}=\mathcal{E}\left(\psi_{y}^{x_{0}}\right)$ (recall that $\psi_{y}^{x_{0}}(y)=0$ and $\psi_{y}^{x_{0}}\left(x_{0}\right)=1$ ). It follows from (A2) that

$$
\begin{aligned}
\psi_{y}^{x_{0}}(x)^{2} & =\left|\psi_{y}^{x_{0}}(x)-\psi_{y}^{x_{0}}(y)\right|^{2} \\
& \leqslant R(x, y) \mathcal{E}\left(\psi_{y}^{x_{0}}\right) \\
& =R(x, y) R\left(x_{0}, y\right)^{-1} \\
& \leqslant c\left(d(y, x) r^{-1}\right)^{\gamma} \leqslant \frac{1}{4}
\end{aligned}
$$

if $d(x, y) \leqslant \varepsilon_{1} r$, where $\varepsilon_{1}$ is a small constant independent of $x_{0}, x, y$ and $r$. Thus, $\psi_{y}^{x_{0}}(x) \leqslant \frac{1}{2}$ for $x \in B\left(y, \varepsilon_{1} r\right)$. We cover $D$ by $N$ balls $\left\{B\left(y_{i}, \varepsilon_{1} r\right)\right\}_{i=1}^{N}$. Since $\mu$ satisfies the doubling condition, the number $N$ can be chosen independent of $x_{0}, y_{i}$ and $r$. Define

$$
v_{0}(x)=\min _{1 \leqslant i \leqslant N} \psi_{y_{i}}^{x_{0}}(x), \quad x \in X
$$

We see that $v_{0}\left(x_{0}\right)=1$ since $\psi_{y_{i}}^{x_{0}}\left(x_{0}\right)=1$, and $v_{0} \leqslant \frac{1}{2}$ on $D$ since $\psi_{y_{i}}^{x_{0}} \leqslant \frac{1}{2}$ on $B\left(y_{i}, \varepsilon_{1} r\right)$, for $1 \leqslant i \leqslant N$. We claim that there exists some $c>0$ independent of $r$ and $x_{0}$ such that

$$
\mathcal{E}\left(v_{0}\right) \leqslant c r^{-\gamma}
$$

In fact, from (5.16), for $x, z \in X$, we have

$$
\left|v_{0}(x)-v_{0}(z)\right| \leqslant \max _{1 \leqslant i \leqslant N}\left|\psi_{y_{i}}^{x_{0}}(x)-\psi_{y_{i}}^{x_{0}}(z)\right| \leqslant \sum_{i=1}^{N}\left|\psi_{y_{i}}^{x_{0}}(x)-\psi_{y_{i}}^{x_{0}}(z)\right| .
$$

Therefore, using (A2),

$$
\begin{aligned}
\mathcal{E}\left(v_{0}\right) & =\lim _{t \rightarrow 0} \frac{1}{2 t} \int_{X} \int_{X}\left(v_{0}(x)-v_{0}(z)\right)^{2} p(t, x, z) \mathrm{d} \mu(z) \mathrm{d} \mu(x) \\
& \leqslant N \sum_{i=1}^{N} \lim _{t \rightarrow 0} \frac{1}{2 t} \int_{X} \int_{X}\left|\psi_{y_{i}}^{x_{0}}(x)-\psi_{y_{i}}^{x_{0}}(z)\right|^{2} p(t, x, z) \mathrm{d} \mu(z) \mathrm{d} \mu(x) \\
& =N \sum_{i=1}^{N} \mathcal{E}\left(\psi_{y_{i}}^{x_{0}}\right) \leqslant N^{2} \max _{1 \leqslant i \leqslant N} R\left(x_{0}, y_{i}\right)^{-1} \leqslant c r^{-\gamma}
\end{aligned}
$$




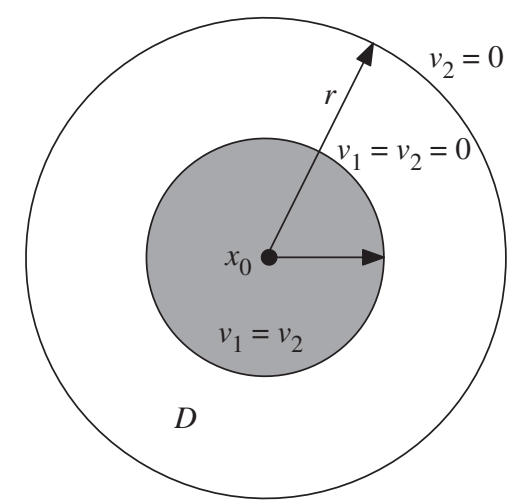

Figure 1. Functions $v_{1}$ and $v_{2}$.

proving (5.17). Let $v_{1}=2\left(v_{0}-\frac{1}{2}\right)_{+}$and $v_{2}(\cdot)=v_{1}(\cdot) \mathbf{1}_{B\left(x_{0}, r\right)}(\cdot)$ (see Figure 1). By the locality of $\mathcal{E}$, we have $\mathcal{E}\left(v_{2}, v_{1}-v_{2}\right)=0$, and so

$$
\mathcal{E}\left(v_{2}\right)=\mathcal{E}\left(v_{1}\right)-\mathcal{E}\left(v_{1}-v_{2}\right)-2 \mathcal{E}\left(v_{2}, v_{1}-v_{2}\right) \leqslant \mathcal{E}\left(v_{1}\right) .
$$

Noting that $v_{2}\left(x_{0}\right)=1$, and $v_{2}=0$ on $B\left(x_{0}, r\right)^{\mathrm{c}}$, we see from (5.17) that

$$
R\left(x_{0}, B\left(x_{0}, r\right)^{\mathrm{c}}\right)^{-1} \leqslant \mathcal{E}\left(v_{2}\right) \leqslant \mathcal{E}\left(v_{1}\right) \leqslant 4 \mathcal{E}\left(v_{0}\right) \leqslant c r^{-\gamma},
$$

proving the lemma.

For $x_{0} \in X$ and $0<r<r_{0}$, define $B:=B\left(x_{0}, r\right)$. Let $\varphi_{0}$ be a continuous function on $X$ satisfying the condition that $0 \leqslant \varphi_{0} \leqslant 1$, and

$$
\varphi_{0}= \begin{cases}1 & \text { on } B\left(x_{0}, \frac{1}{2} r\right), \\ 0 & \text { on } B\left(x_{0}, \frac{2}{3} r\right)^{\mathrm{c}} .\end{cases}
$$

Consider the Poisson equation with zero boundary condition

$$
\begin{aligned}
-H_{B} u_{0}=\varphi_{0} & \text { in } B, \\
u_{0}=0 & \text { in } B^{\mathrm{c}},
\end{aligned}
$$

where $H_{B}$ is the generator of the Dirichlet form $\left(\mathcal{E}, \mathcal{F}_{B}\right)$. We say that a function $u_{0}$ defined on $X$ is a weak solution to (5.20), (5.21) if $u_{0} \in \mathcal{F}_{B}$ and

$$
\mathcal{E}\left(u_{0}, v\right)=\int_{B} v(x) \varphi_{0}(x) \mathrm{d} \mu(x)
$$

for any $v \in \mathcal{F}_{B}$. Note that a weak solution does not necessarily belong to the domain $\mathcal{D}\left(H_{B}\right)$ of $H_{B}$. If it does, and (5.20) holds pointwise in $B$, we call it a strong solution. Equation (5.20) with (5.21) has a unique weak solution,

$$
u_{0}(x)=\int_{X} g_{B^{\mathrm{c}}}^{x}(y) \varphi_{0}(y) \mathrm{d} \mu(y)=\int_{B} g_{B^{\mathrm{c}}}^{x}(y) \varphi_{0}(y) \mathrm{d} \mu(y), \quad x \in X,
$$


where $g_{B^{\mathrm{c}}}^{x}(\cdot)=R\left(x, B^{\mathrm{c}}\right) \psi_{B^{\mathrm{c}}}^{x}(\cdot)$ is the Green function whose existence was proved in Proposition 4.2. In fact, from (5.23) and (4.5), for any $v \in \mathcal{F}_{B}$, we have

$$
\begin{aligned}
\mathcal{E}\left(u_{0}, v\right) & =\lim _{t \rightarrow 0} \frac{1}{t} \int_{X}\left(u_{0}(x)-T_{t} u_{0}(x)\right) v(x) \mathrm{d} \mu(x) \\
& =\lim _{t \rightarrow 0} \frac{1}{t} \int_{X}\left(\int_{X}\left(g_{B^{c}}^{y}(x)-\left(T_{t} g_{B^{c}}^{y}\right)(x)\right) v(x) \mathrm{d} \mu(x)\right) \varphi_{0}(y) \mathrm{d} \mu(y) \\
& =\lim _{t \rightarrow 0} \int_{X} \mathcal{E}_{t}\left(g_{B^{c}}^{y}, v\right) \varphi_{0}(y) \mathrm{d} \mu(y) \\
& =\int_{X} \mathcal{E}\left(g_{B^{c}}^{y}, v\right) \varphi_{0}(y) \mathrm{d} \mu(y) \\
& =\int_{B} v(y) \varphi_{0}(y) \mathrm{d} \mu(y),
\end{aligned}
$$

and so $u_{0}$ is a weak solution to $(5.20),(5.21)$. The uniqueness easily follows from the irreducibility of $\mathcal{E}$.

Alternatively, the Green function $g_{B^{c}}^{x}$ above may usefully be expressed as

$$
g_{B^{\mathrm{c}}}^{x}(y)=\int_{0}^{\infty} p_{B}(t, x, y) \mathrm{d} t, \quad x, y \in B
$$

where $p_{B}(t, x, y)$ is the heat kernel of $\left(\mathcal{E}, \mathcal{F}_{B}\right)$. (Note that $p_{B}(t, x, y)$ exists, and is continuous on $(0, \infty) \times B \times B$. The finiteness of the integral in (5.25) may be seen below.) In other words, the solution $u_{0}$ may also be written as

$$
u_{0}(x)=\int_{B}\left(\int_{0}^{\infty} p_{B}(t, x, y) \mathrm{d} t\right) \varphi_{0}(y) \mathrm{d} \mu(y)=\int_{0}^{\infty} T_{t}^{B} \varphi_{0}(x) \mathrm{d} t, \quad x \in X .
$$

In fact, from (5.26) we have

$$
u_{0}(x)-T_{t}^{B} u_{0}(x)=\int_{0}^{t} T_{s}^{B} \varphi_{0}(x) \mathrm{d} s
$$

and so, for $v \in \mathcal{F}_{B}$,

$$
\mathcal{E}\left(u_{0}, v\right)=\lim _{t \rightarrow 0} \frac{1}{t} \int_{B}\left(u_{0}(x)-T_{t}^{B} u_{0}(x)\right) v(x) \mathrm{d} \mu(x)=\int_{B} v(x) \varphi_{0}(x) \mathrm{d} \mu(x) .
$$

Remark 5.4. Let $X_{t}$ be the Hunt process associated with the regular Dirichlet form $(\mathcal{E}, \mathcal{F})$. Then the solution to $(5.20),(5.21)$ can be written as

$$
u_{0}(x)=\mathbb{E}_{x}\left(\int_{0}^{\tau_{B}} \varphi_{0}\left(X_{t}\right) \mathrm{d} t\right) .
$$

In fact, using the Fubini theorem, we see from (5.26) that

$$
\begin{aligned}
u_{0}(x) & =\int_{0}^{\infty} \mathbb{E}_{x}^{B}\left(\varphi_{0}\left(X_{t}\right)\right) \mathrm{d} t \\
& =\int_{0}^{\infty} \mathbb{E}_{x}\left(\mathbf{1}_{\left\{t<\tau_{B}\right\}} \varphi_{0}\left(X_{t}\right)\right) \mathrm{d} t
\end{aligned}
$$




$$
\begin{aligned}
& =\mathbb{E}_{x}\left(\int_{0}^{\infty} \mathbf{1}_{\left\{t<\tau_{B}\right\}} \varphi_{0}\left(X_{t}\right) \mathrm{d} t\right) \\
& =\mathbb{E}_{x}\left(\int_{0}^{\tau_{B}} \varphi_{0}\left(X_{t}\right) \mathrm{d} t\right) .
\end{aligned}
$$

We now estimate $u_{0}$.

Proposition 5.5. Assume that all the hypotheses in Proposition 5.3 hold, and that $\mu$ satisfies (A1). Let $u_{0}$ be the solution to (5.20), (5.21). There then exist constants $c_{3}, c_{4}, \varepsilon_{2}>0$ independent of $x_{0}$ and $r$ such that

$$
c_{3} r^{\beta} \leqslant \min _{x \in B\left(x_{0}, \varepsilon_{2} r\right)} u_{0}(x) \leqslant \max _{x \in X} u_{0}(x) \leqslant c_{4} r^{\beta} .
$$

Proof. The key is to estimate the Green function $g_{B^{c}}^{x}$. This can be done by using (A1) and (A2). Using (4.13) and the upper bound of $R$ in (A2), we have

$$
g_{B^{\mathrm{c}}}^{x}(y) \leqslant g_{B^{\mathrm{c}}}^{x}(x)=R\left(x, B^{\mathrm{c}}\right) \leqslant \inf _{z \in B^{\mathrm{c}}} R(x, z) \leqslant c_{2} r^{\gamma}
$$

for all $x, y \in X$. Thus, it follows from (5.23) and (A1) that, using $\gamma=\beta-\alpha$,

$$
u_{0}(x) \leqslant c_{2} r^{\gamma} \mu\left(B\left(x_{0}, r\right)\right) \leqslant c_{4} r^{\gamma+\alpha}=c_{4} r^{\beta}
$$

for all $x \in X$, proving the third inequality in (5.29). On the other hand, let $\psi_{B^{\mathrm{c}}}^{x}$ be such that $R\left(x, B^{\mathrm{c}}\right)^{-1}=\mathcal{E}\left(\psi_{B^{\mathrm{c}}}^{x}\right)$, for $x \in B\left(x_{0}, \frac{1}{2} r\right)$. It follows from (5.14) and (A2) that, for $x \in B\left(x_{0}, \frac{1}{2} r\right)$,

$$
\begin{aligned}
\left(1-\psi_{B^{\mathrm{c}}}^{x}(y)\right)^{2} & =\left(\psi_{B^{\mathrm{c}}}^{x}(x)-\psi_{B^{\mathrm{c}}}^{x}(y)\right)^{2} \\
& \leqslant R(x, y) \mathcal{E}\left(\psi_{B^{\mathrm{c}}}^{x}\right) \\
& =R(x, y) R\left(x, B^{\mathrm{c}}\right)^{-1} \\
& \leqslant R(x, y) R\left(x, B\left(x, \frac{1}{4} r\right)^{\mathrm{c}}\right)^{-1} \\
& \leqslant c\left(d(x, y) r^{-1}\right)^{\gamma} \\
& \leqslant \frac{1}{4},
\end{aligned}
$$

if $y \in B\left(x, 2 \varepsilon_{2} r\right)$ for a small $\varepsilon_{2} \in\left(0, \frac{1}{2}\right)$ independent of $x_{0}$ and $r$. Thus, $\psi_{B^{\mathrm{c}}}^{x}(y) \geqslant \frac{1}{2}$. Therefore, for all $x, y \in B\left(x_{0}, \varepsilon_{2} r\right)$,

$$
g_{B^{\mathrm{c}}}^{x}(y)=R\left(x, B^{\mathrm{c}}\right) \psi_{B^{\mathrm{c}}}^{x}(y) \geqslant \frac{1}{2} R\left(x, B\left(x, \frac{1}{4} r\right)^{\mathrm{c}}\right) \geqslant c r^{\gamma},
$$

where $c$ is independent of $x_{0}$ and $r$. Hence, from (5.23) and (A1) we have

$$
u_{0}(x)=\int_{B} g_{B^{\mathrm{c}}}^{x}(y) \varphi_{0}(y) \mathrm{d} \mu(y) \geqslant \int_{B\left(x_{0}, \varepsilon_{2} r\right)} g_{B^{\mathrm{c}}}^{x}(y) \mathrm{d} \mu(y) \geqslant c_{3} r^{\beta},
$$

for all $x \in B\left(x_{0}, \varepsilon_{2} r\right)$. This proves the first inequality in (5.29). 
Remark 5.6. By Remark 5.4, the estimates in (5.29) may be obtained by estimating $\mathbb{E}_{x}\left(\tau_{B}\right)$ and $\mathbb{E}_{x}\left(\tau_{B\left(x_{0}, r / 2\right)}\right)$ by using the fact that

$$
\mathbb{E}_{x}\left(\tau_{B\left(x_{0}, r / 2\right)}\right) \leqslant u_{0}(x) \leqslant \mathbb{E}_{x}\left(\tau_{B}\right), \quad x \in B .
$$

For $x_{0} \in X$ and $0<r<r_{0}$, let $B=B\left(x_{0}, r\right)$ as before. The estimates in (5.29) give rise to a lower integral estimate for the heat kernel $p_{B}(t, x, y)$, that is, there exist two constants $c_{5} \in(0,1)$ and $c_{6}>0$ independent of $x_{0}, r$ and $t$ such that, for all $x \in B\left(x_{0}, \varepsilon_{2} r\right)$ and $t>0$,

$$
\int_{B} p_{B}(t, x, y) \mathrm{d} \mu(y) \geqslant c_{5}-c_{6} r^{-\beta} t .
$$

Indeed, let $u_{0}$ be the solution to (5.20), (5.21). Using the semigroup property, we see from (5.26) that, for $t>0$,

$$
\begin{aligned}
\int_{t}^{\infty}\left(\int_{B} p_{B}(s, x, y)\right. & \left.\varphi_{0}(y) \mathrm{d} \mu(y)\right) \mathrm{d} s \\
& =\int_{0}^{\infty}\left(\int_{B} p_{B}(s+t, x, y) \varphi_{0}(y) \mathrm{d} \mu(y)\right) \mathrm{d} s \\
& =\int_{0}^{\infty}\left(\int_{B}\left\{\int_{B} p_{B}(t, x, z) p_{B}(s, z, y) \mathrm{d} \mu(z)\right\} \varphi_{0}(y) \mathrm{d} \mu(y)\right) \mathrm{d} s \\
& =\int_{B} u_{0}(z) p_{B}(t, x, z) \mathrm{d} \mu(z) \\
& \leqslant \max _{z \in B} u_{0}(z) \int_{B} p_{B}(t, x, z) \mathrm{d} \mu(z) .
\end{aligned}
$$

Therefore, noting that

$$
\int_{B} p_{B}(s, x, y) \mathrm{d} \mu(y) \leqslant 1 \text { and } 0 \leqslant \varphi_{0} \leqslant 1
$$

we have

$$
\begin{aligned}
u_{0}(x) & =\int_{0}^{\infty}\left(\int_{B} p_{B}(s, x, y) \varphi_{0}(y) \mathrm{d} \mu(y)\right) \mathrm{d} s \\
& =\int_{0}^{t}\left(\int_{B} p_{B}(s, x, y) \varphi_{0}(y) \mathrm{d} \mu(y)\right) \mathrm{d} s+\int_{t}^{\infty}\left(\int_{B} p_{B}(s, x, y) \varphi_{0}(y) \mathrm{d} \mu(y)\right) \mathrm{d} s \\
& \leqslant t+\max _{z \in B} u_{0}(z) \int_{B} p_{B}(t, x, z) \mathrm{d} \mu(z),
\end{aligned}
$$

which yields

$$
\int_{B} p_{B}(t, x, z) \mathrm{d} \mu(z) \geqslant \frac{u_{0}(x)-t}{\max u_{0}}, \quad x \in X, t>0 .
$$


Thus, (5.33) follows by using (5.29). Combining (5.33) and (2.11), we see that

$$
\begin{aligned}
\int_{B\left(x_{0}, r\right)^{\mathrm{c}}} p(t, x, y) \mathrm{d} \mu(y) & =1-\int_{B} p(t, x, y) \mathrm{d} \mu(y) \\
& \leqslant 1-\int_{B} p_{B}(t, x, y) \mathrm{d} \mu(y) \leqslant \varepsilon+c_{6} r^{-\beta} t
\end{aligned}
$$

for all $t>0$ and $x \in B\left(x_{0}, \varepsilon_{2} r\right)$, where $\varepsilon=1-c_{5} \in(0,1)$.

Remark 5.7. From the viewpoint of probability theory, we have

$$
\int_{B\left(x_{0}, r\right)^{\mathrm{c}}} p(t, x, y) \mathrm{d} \mu(y)=T_{t} \mathbf{1}_{B^{\mathrm{c}}}(x)=\mathbb{P}_{x}\left(X_{t} \in B^{\mathrm{c}}\right) \leqslant \mathbb{P}_{x}\left(\tau_{B} \leqslant t\right) .
$$

Thus, (5.35) can be obtained by estimating $\mathbb{P}_{x}\left(\tau_{B} \leqslant t\right)$. The reader may consult $[\mathbf{2}$, Lemma 3.16, p. 33] for the estimates on $\mathbb{P}_{x}\left(\tau_{B} \leqslant t\right)$ for the special case $x=x_{0}$ when assuming that $\mathbb{E}_{y}\left(\tau_{B(y, r)}\right) \asymp r^{\beta}$ for any $y \in X$.

Remark 5.8. The estimate (5.33) is interesting. To see this, note that, for any $\lambda>0$ and $x \in X$,

$$
\begin{aligned}
\lambda \int_{0}^{\infty} \mathrm{e}^{-\lambda t} T_{t}^{B} 1(x) \mathrm{d} t & =\lambda \int_{0}^{\infty} \mathrm{e}^{-\lambda t} \mathbb{E}_{x}\left(\mathbf{1}_{\left\{t<\tau_{B}\right\}}\right) \mathrm{d} t \\
& =\mathbb{E}_{x}\left(\int_{0}^{\tau_{B}} \lambda \mathrm{e}^{-\lambda t} \mathrm{~d} t\right) \\
& =1-\mathbb{E}_{x}\left(\mathrm{e}^{-\lambda \tau_{B}}\right) .
\end{aligned}
$$

On the other hand, we see from (5.33) that, for all $x \in B\left(x_{0}, \varepsilon_{2} r\right)$,

$$
\begin{aligned}
\lambda \int_{0}^{\infty} \mathrm{e}^{-\lambda t} T_{t}^{B} 1(x) \mathrm{d} t & =\lambda \int_{0}^{\infty} \mathrm{e}^{-\lambda t}\left(\int_{B} p_{B}(t, x, y) \mathrm{d} \mu(y)\right) \mathrm{d} t \\
& \geqslant \lambda \int_{0}^{\infty} \mathrm{e}^{-\lambda t}\left(c_{5}-c_{6} r^{-\beta} t\right) \mathrm{d} t \\
& =c_{5}-c\left(\lambda r^{\beta}\right)^{-1}
\end{aligned}
$$

Combining (5.37) and (5.38), we obtain

$$
\mathbb{E}_{x}\left(\mathrm{e}^{-\lambda \tau_{B}}\right)=1-\lambda \int_{0}^{\infty} \mathrm{e}^{-\lambda t} T_{t}^{B} 1(x) \mathrm{d} t \leqslant\left(1-c_{5}\right)+c\left(\lambda r^{\beta}\right)^{-1}
$$

for all $x \in B\left(x_{0}, \varepsilon_{2} r\right)$ and $\lambda>0$.

Estimate (5.35) is useful, but it is not good enough to obtain optimal off-diagonal upper bounds of $p(t, x, y)$. We need a more delicate estimate than (5.35) (see (5.55), below). In order to do this, we first estimate the solution to an elliptic equation in the ball $B=B\left(x_{0}, r\right)$. Let $\Delta$ be defined as in $(2.7)$. For $\lambda>0$, consider a function $u_{1} \in \mathcal{D}(\Delta)$ satisfying the equation

$$
\Delta u_{1}=\lambda u_{1} \quad \text { in } B
$$


Here $u_{1}$ is a strong solution, that is $u_{1} \in \mathcal{D}(\Delta)$ satisfies (5.40) pointwise in $B$. The solution $u_{1}$ of $(5.40)$ exists, which coincides in $B\left(x_{0}, \frac{1}{2} r\right)$ with $u_{\lambda}$ determined by $(5.52)$ or $(5.53)$, below.

Lemma 5.9. Assume that all the hypotheses (A1), (A2) and (2.16) hold. Let $u_{1}$ satisfy (5.40). If $0 \leqslant u_{1} \leqslant 1$ on $\bar{B}$, then there exists some $\varepsilon_{3} \in(0,1)$ independent of $x_{0}$, $r$ and $\lambda$ such that

$$
u_{1}(x) \leqslant \varepsilon_{3}
$$

for all $x \in B\left(x_{0}, \varepsilon_{2} r\right)$, provided that $\lambda r^{\beta}$ is sufficiently large.

Proof. Set $u=1-u_{1}$. Then $u$ satisfies

$$
(\lambda-\Delta) u=\lambda \quad \text { in } B
$$

Let $\varphi_{0}$ be as before; see (5.19). Let $v \in \mathcal{F}_{B}$ be the solution to the equation

$$
\left(\lambda-\Delta_{B}\right) v=\lambda \varphi_{0} \quad \text { in } B
$$

where $\Delta_{B}$ is defined in the same way as in (2.7) for the semigroup $\left\{T_{t}^{B}\right\}$ of the form $\left(\mathcal{E}, \mathcal{F}_{B}\right)$. It is easy to see that

$$
v(x)=\lambda \int_{0}^{\infty} \mathrm{e}^{-\lambda t} T_{t}^{B} \varphi_{0}(x) \mathrm{d} t, \quad x \in B .
$$

Note that $v$ is a strong solution to (5.43), that is, the function $v \in \mathcal{D}\left(\Delta_{B}\right)$ satisfies (5.43) pointwise in $B$. Indeed, one can easily verify from (5.44) that

$$
\lim _{t \rightarrow 0}\left\|t^{-1}\left(T_{t}^{B} v-v\right)-\lambda\left(v-\varphi_{0}\right)\right\|_{C(B)}=0
$$

by using $(5.13)$, and so $\Delta_{B} v=\lambda\left(v-\varphi_{0}\right)$ in $B$. Now we claim that

$$
v(x) \leqslant u(x), \quad x \in \bar{B} .
$$

Indeed, letting $h=u-v$, we see that $h$ is continuous on $\bar{B}$ since both $u$ and $v$ are continuous on $\bar{B}$. Since $\left.h\right|_{\partial B}=\left.u\right|_{\partial B} \geqslant 0$, the inequality (5.45) holds if we can show that $h \geqslant 0$ in $B$. Assume that this is not true. Then there would be a point $y_{0} \in B$ such that $h\left(y_{0}\right)=\min _{\bar{B}} h<0$. Let $\delta>0$ be so small that $B\left(y_{0}, \delta\right) \subset B$. Thus, it follows from $(2.17)$ that

$$
\begin{aligned}
\lim _{t \rightarrow 0} \frac{1}{t}\left(T_{t} h\left(y_{0}\right)-h\left(y_{0}\right)\right) & =\lim _{t \rightarrow 0} \frac{1}{t}\left\{\int_{B\left(y_{0}, \delta\right)}\left(h(y)-h\left(y_{0}\right)\right) p\left(t, y_{0}, y\right) \mathrm{d} \mu(y)\right. \\
& \geqslant \lim _{t \rightarrow 0} \frac{1}{t} \int_{B\left(y_{0}, \delta\right)^{\mathrm{c}}}\left(h(y)-h\left(y_{0}\right)\right) p\left(t, y_{0}, y\right) \mathrm{d} \mu(y) \\
& =0 .
\end{aligned}
$$


Therefore, by (5.42) and (5.43) and using (2.11),

$$
\begin{aligned}
0 & >\lambda h\left(y_{0}\right)=\lambda\left(1-\varphi_{0}\left(y_{0}\right)\right)+\Delta u\left(y_{0}\right)-\Delta_{B} v\left(y_{0}\right) \\
& \geqslant \lim _{t \rightarrow 0} \frac{T_{t} u\left(y_{0}\right)-u\left(y_{0}\right)-\left(T_{t}^{B} v\left(y_{0}\right)-v\left(y_{0}\right)\right)}{t} \\
& \geqslant \lim _{t \rightarrow 0} \frac{T_{t} h\left(y_{0}\right)-h\left(y_{0}\right)}{t} \geqslant 0
\end{aligned}
$$

which is a contradiction. This proves (5.45). Finally, by (5.33) with $B$ replaced by $B\left(x_{0}, \frac{1}{2} r\right)$,

$$
\begin{aligned}
T_{t}^{B} \varphi_{0}(x) & =\int_{B} p_{B}(t, x, y) \varphi_{0}(y) \mathrm{d} \mu(y) \\
& \geqslant \int_{B\left(x_{0}, r / 2\right)} p_{B\left(x_{0}, r / 2\right)}(t, x, y) \mathrm{d} \mu(y) \geqslant c_{5}-2^{\beta} c_{6} r^{-\beta} t
\end{aligned}
$$

and so

$$
v(x)=\lambda \int_{0}^{\infty} \mathrm{e}^{-\lambda t} T_{t}^{B} \varphi_{0}(x) \mathrm{d} t \geqslant c_{5}-c\left(r^{\beta} t\right)^{-1} .
$$

This combines with (5.45), for all $x \in B\left(x_{0}, \varepsilon_{2} r\right)$, to give

$$
u_{1}(x)=1-u(x) \leqslant 1-v(x) \leqslant\left(1-c_{5}\right)+c\left(\lambda r^{\beta}\right)^{-1} \leqslant \varepsilon_{3},
$$

if $\lambda r^{\beta}$ is sufficiently large.

The estimate (5.41) will give a more accurate bound of $u_{1}$ at the point $x_{0}$.

Lemma 5.10. Assume that the hypotheses in Lemma 5.9 hold. There then exist $c, c_{7}>0$ independent of $x_{0}, r$ and $\lambda$ such that

$$
u_{1}\left(x_{0}\right) \leqslant c \exp \left(-c_{7} \lambda^{1 / \beta} r\right)
$$

Proof. We consider only the case where $\lambda r^{\beta}$ is large; otherwise, (5.48) is trivial since $u_{1} \leqslant 1$ on $\bar{B}$. Let $r^{\prime}=r / n$, where $n \geqslant 2$ is an integer to be determined below. Denote by $x_{i}$ the maximum point of $u_{1}$ on the closed ball $\bar{B}\left(x_{0}, i r^{\prime}\right)$ for $1 \leqslant i \leqslant n$ (since $u_{1}$ is continuous, each of such points $x_{i}$ exists). Set $m_{i}=u_{1}\left(x_{i}\right)$ for $1 \leqslant i \leqslant n$.

Consider the ball $B_{i}:=B\left(x_{i}, \frac{1}{2} r^{\prime}\right)$. For $1 \leqslant i \leqslant n-1$, define

$$
v_{i}(x)=\frac{u_{1}(x)}{m_{i+1}} \quad \text { for } x \in X .
$$

Then $v_{i}$ satisfies the condition that $\Delta v_{i}=\lambda v_{i}$ in $B_{i}$ (see Figure 2).

By Lemma 5.9 with $x_{0}$ replaced by $x_{i}$ and $r$ by $\frac{1}{2} r^{\prime}$, and noting that $0 \leqslant v_{i} \leqslant 1$ on $\bar{B}_{i}$, we find that $v_{i}\left(x_{i}\right) \leqslant \varepsilon_{3}$ if $\lambda\left(\frac{1}{2} r^{\prime}\right)^{\beta} \geqslant c$ for some large $c$. Therefore,

$$
m_{i} \leqslant \varepsilon_{3} m_{i+1}, \quad 1 \leqslant i \leqslant n-1,
$$




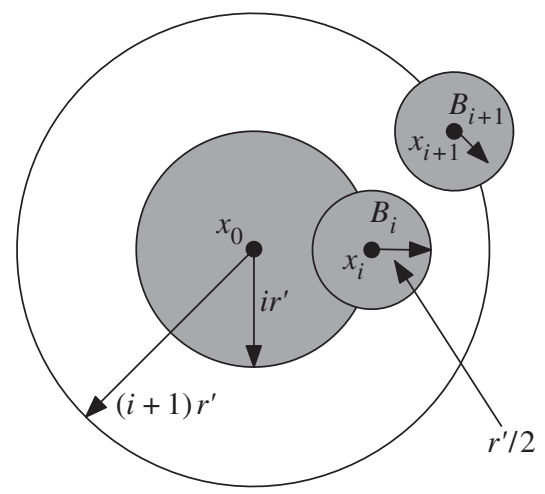

Figure 2. $\Delta v_{i}=\lambda v_{i}$ in $B_{i}$, and $0 \leqslant v_{i} \leqslant 1$ on $\bar{B}_{i}$.

which gives

$$
m_{1} \leqslant \varepsilon_{3}^{n-1} m_{n} \leqslant \varepsilon_{3}^{n-1}
$$

by noting that $m_{n} \leqslant 1$. Setting $c^{\prime}=-\log \varepsilon_{3}>0$, we see that

$$
u_{1}\left(x_{0}\right) \leqslant m_{1}=u_{1}\left(x_{1}\right) \leqslant \exp \left(-c^{\prime}(n-1)\right) \leqslant \exp \left(-c_{7} \lambda^{1 / \beta} r\right),
$$

by choosing the largest integer $n$ so that $\lambda\left(\frac{1}{2} r^{\prime}\right)^{\beta} \geqslant c$. Therefore, (5.48) follows.

The proof of Lemma 5.10 given here is motivated by [15, Lemma 5.4] (see also [14, Lemma 7.3]) on infinite graphs.

Remark 5.11. It follows from (5.39) that

$$
\mathbb{E}_{x_{0}}\left(\mathrm{e}^{-\lambda \tau_{B}}\right) \leqslant \varepsilon_{3}
$$

if $\lambda r^{\beta}$ is sufficiently large. By the locality of $\mathcal{E}$ and using the chain argument (see [2, pp. 34, $35]$ or $[\mathbf{1 3}$, Theorem 9.1 , (iv) $\Rightarrow(\mathrm{v})]$ ), one can find from (5.49) that

$$
\mathbb{E}_{x_{0}}\left(\mathrm{e}^{-\lambda \tau_{B}}\right) \leqslant c \exp \left(-c_{7} \lambda^{1 / \beta} r\right)
$$

by applying the strong Markov property of the diffusion $\left(\left\{X_{t}\right\}_{t \geqslant 0},\left\{\mathbb{P}_{x}\right\}_{x \in X}\right)$ of the form $(\mathcal{E}, \mathcal{F})$. On the other hand, letting $B_{1}=B\left(x_{0}, \frac{1}{2} r\right)$, we see from $(5.37)$ that

$$
v\left(x_{0}\right)=\lambda \int_{0}^{\infty} \mathrm{e}^{-\lambda t} T_{t}^{B} \varphi_{0}\left(x_{0}\right) \mathrm{d} t \geqslant \lambda \int_{0}^{\infty} \mathrm{e}^{-\lambda t} T_{t}^{B_{1}} 1\left(x_{0}\right) \mathrm{d} t=1-\mathbb{E}_{x_{0}}\left(\mathrm{e}^{-\lambda \tau_{B_{1}}}\right) .
$$

Thus, combining (5.47) and (5.50) with $r$ replaced by $\frac{1}{2} r$, it follows that

$$
u_{1}\left(x_{0}\right)=1-u\left(x_{0}\right) \leqslant 1-v\left(x_{0}\right) \leqslant \mathbb{E}_{x_{0}}\left(\mathrm{e}^{-\lambda \tau_{B_{1}}}\right) \leqslant c \exp \left(-c^{\prime} \lambda^{1 / \beta} r\right) .
$$

Hence, (5.48) can be also obtained by using probability theory. 
To obtain the key estimate (5.55) below, we introduce a function $u_{\lambda}$ on $X$ determined by the equation

$$
(\lambda-\Delta) u_{\lambda}=\lambda \varphi_{1} \quad \text { on } X
$$

for $\lambda>0$, where $0 \leqslant \varphi_{1} \leqslant 1$ is a continuous function on $X$ satisfying $\varphi_{1}(x)=1$ for $x \in B\left(x_{0}, \frac{2}{3} r\right)^{\mathrm{c}}$ and $\varphi_{1}(x)=0$ for $x \in B\left(x_{0}, \frac{1}{2} r\right)$. Observe that (5.52) has a unique strong solution

$$
u_{\lambda}(x)=\lambda \int_{0}^{\infty} \exp (-\lambda t) T_{t} \varphi_{1}(x) \mathrm{d} t, \quad x \in X,
$$

for any $\lambda>0$, by using (5.8). By (5.53), we have $0 \leqslant u_{\lambda} \leqslant 1$ on $X$. Note that, for $x \in B\left(x_{0}, r\right)^{\mathrm{c}}$, using (5.33) with $x_{0}$ replaced by $x$, we have

$$
\begin{aligned}
T_{t} \varphi_{1}(x) & \geqslant \int_{B\left(x_{0}, 2 r / 3\right)^{\mathrm{c}}} p(t, x, y) \mathrm{d} \mu(y) \\
& \geqslant \int_{B(x, r / 3)} p(t, x, y) \mathrm{d} \mu(y) \\
& \geqslant \int_{B(x, r / 3)} p_{B(x, r / 3)}(t, x, y) \mathrm{d} \mu(y) \\
& \geqslant c_{5}-c_{6}\left(\frac{1}{3} r\right)^{-\beta} t \geqslant \frac{1}{2} c_{5}
\end{aligned}
$$

if $r^{\beta} t^{-1} \geqslant c$ for a large $c$. Thus, from (5.53) we have

$$
u_{\lambda}(x) \geqslant \lambda \int_{0}^{c r^{\beta}} \mathrm{e}^{-\lambda t} T_{t} \varphi_{1}(x) \mathrm{d} t \geqslant \frac{1}{2} c_{5}\left(1-\mathrm{e}^{-c \lambda r^{\beta}}\right) \geqslant \frac{1}{4} c_{5}
$$

if $\lambda r^{\beta}$ is large. Therefore,

$$
u_{\lambda}(x) \geqslant \frac{1}{4} c_{5} \mathbf{1}_{B\left(x_{0}, r\right)^{\mathrm{c}}}(x)
$$

for all $x \in X$ if $\lambda r^{\beta}$ is large.

Theorem 5.12. Let $(X, d, \mu)$ be a metric measure space satisfying the chain condition, and let $(\mathcal{E}, \mathcal{F})$ be a Dirichlet form. Assume that the hypotheses (A1), (A2) and (2.16) all hold. Then, for $x_{0} \in X$ and $r \in\left(0, r_{0}\right)$,

$$
\int_{B\left(x_{0}, r\right)^{\mathrm{c}}} p\left(t, x_{0}, y\right) \mathrm{d} \mu(y) \leqslant c \exp \left(-c_{8}\left(r^{\beta} t^{-1}\right)^{1 /(\beta-1)}\right),
$$

where $c, c_{8}$ are independent of $x_{0}, r$ and $t$.

Proof. Let $u_{\lambda}$ be as in (5.52). Observe that $u_{\lambda} \in \mathcal{D}(\Delta)$ satisfies

$$
\Delta u_{\lambda}=\lambda u_{\lambda} \quad \text { in } B\left(x_{0}, \frac{1}{2} r\right) .
$$

Thus, it follows from (5.48) that

$$
u_{\lambda}\left(x_{0}\right) \leqslant c \exp \left(-c_{7} \lambda^{1 / \beta} r\right)
$$


Define

$$
w_{\lambda}(t, x)=\mathrm{e}^{\lambda t} u_{\lambda}(x), \quad t>0, x \in X .
$$

Clearly, for $x \in X$ and $t>0$,

$$
\frac{\partial w_{\lambda}}{\partial t}(t, x)=\lambda \mathrm{e}^{\lambda t} u_{\lambda}(x)=\Delta w_{\lambda}(x)+\lambda \mathrm{e}^{\lambda t} \varphi_{1}(x)
$$

with initial value $w_{\lambda}(0, x)=u_{\lambda}(x)(x \in X)$. Thus, we see that, using (5.54),

$$
\begin{aligned}
w_{\lambda}(t, x) & =\int_{X} u_{\lambda}(y) p(t, x, y) \mathrm{d} \mu(y)+\lambda \int_{0}^{t} \mathrm{e}^{\lambda s} \mathrm{~d} s \int_{X} \varphi_{1}(y) p(t-s, x, y) \mathrm{d} \mu(y) \\
& \geqslant \frac{1}{4} c_{5} \int_{B\left(x_{0}, r\right)^{\mathrm{c}}} p(t, x, y) \mathrm{d} \mu(y)
\end{aligned}
$$

for $t>0, x \in X$ and any $\lambda>0$, if $\lambda r^{\beta} \geqslant c$ for a large $c$. For a suitable $\lambda$, we need to bound $w_{\lambda}\left(t, x_{0}\right)$ for $t>0$. Indeed, from (5.57) and (5.56) we obtain

$$
w_{\lambda}\left(t, x_{0}\right) \leqslant c \exp \left(\lambda t-c_{7} \lambda^{1 / \beta} r\right) \leqslant c \exp \left(-c_{7}\left(t^{-1} r^{\beta}\right)^{1 /(\beta-1)}\right),
$$

by letting $2 \lambda t=c_{7} \lambda^{1 / \beta} r$, if $t^{-1} r^{\beta} \geqslant c$. Thus, it follows from (5.58) that

$$
\int_{B\left(x_{0}, r\right)^{\mathrm{c}}} p\left(t, x_{0}, y\right) \mathrm{d} \mu(y) \leqslant \frac{4}{c_{5}} w_{\lambda}\left(t, x_{0}\right) \leqslant c \exp \left(-c_{7}\left(t^{-1} r^{\beta}\right)^{1 /(\beta-1)}\right),
$$

giving (5.55), if $t^{-1} r^{\beta} \geqslant c$ for a large $c$. However, if $t^{-1} r^{\beta} \leqslant c$, then (5.55) is obvious.

Remark 5.13. Note that from (5.50) one can easily obtain

$$
\begin{aligned}
\mathbb{P}_{x_{0}}\left(\tau_{B} \leqslant t\right) & \leqslant \mathrm{e}^{\lambda t} \mathbb{E}_{x_{0}}\left(\mathrm{e}^{-\lambda \tau_{B}}\right) \\
& \leqslant c \exp \left(\lambda t-c_{7} \lambda^{1 / \beta} r\right) \\
& \leqslant c \exp \left(-c_{7}\left(t^{-1} r^{\beta}\right)^{1 /(\beta-1)}\right)
\end{aligned}
$$

by choosing a suitable $\lambda$, as above. Thus, (5.55) can alternatively be obtained in this way, by noting the fact that

$$
\int_{B^{\mathrm{c}}} p\left(t, x_{0}, y\right) \mathrm{d} \mu(y)=\mathbb{P}_{x_{0}}\left(X_{t} \in B^{\mathrm{c}}\right) \leqslant \mathbb{P}_{x_{0}}\left(\tau_{B} \leqslant t\right) .
$$

We are now in a position to derive off-diagonal upper bounds of $p(t, x, y)$ by using (5.1) and (5.55). Fix $x_{0}, y_{0} \in X, x_{0} \neq y_{0}$, and let $r=\frac{1}{2} d\left(x_{0}, y_{0}\right)$. By (5.1) and (5.55), we have

$$
\begin{aligned}
\int_{B\left(x_{0}, r\right)^{\mathrm{c}}} p\left(t, x_{0}, z\right) p\left(t, z, y_{0}\right) \mathrm{d} \mu(z) & \leqslant c t^{-\alpha / \beta} \int_{B\left(x_{0}, r\right)^{\mathrm{c}}} p\left(t, x_{0}, z\right) \mathrm{d} \mu(z) \\
& \leqslant c t^{-\alpha / \beta} \exp \left(-c^{\prime}\left(t^{-1} d\left(y_{0}, x_{0}\right)^{\beta}\right)^{1 /(\beta-1)}\right) .
\end{aligned}
$$


The above estimate is true if we exchange $x_{0}$ and $y_{0}$. Therefore, by the semigroup property of $p(t, x, y)$, it follows that

$$
\begin{aligned}
p\left(2 t, x_{0}, y_{0}\right) & =\int_{X} p\left(t, x_{0}, z\right) p\left(t, z, y_{0}\right) \mathrm{d} \mu(z) \\
& \leqslant \int_{B\left(x_{0}, r\right)^{\mathrm{c}}} p\left(t, x_{0}, z\right) p\left(t, z, y_{0}\right) \mathrm{d} \mu(z)+\int_{B\left(y_{0}, r\right)^{\mathrm{c}}} p\left(t, x_{0}, z\right) p\left(t, z, y_{0}\right) \mathrm{d} \mu(z) \\
& \leqslant c t^{-\alpha / \beta} \exp \left(-c^{\prime}\left(t^{-1} d\left(y_{0}, x_{0}\right)^{\beta}\right)^{1 /(\beta-1)}\right),
\end{aligned}
$$

giving the upper bound of $p(t, x, y)$ in (A3) for $0<t \leqslant r_{0}$.

\subsection{Off-diagonal lower bounds}

Lower bounds of $p(t, x, y)$ can be obtained in a standard way. We sketch the proof, as follows. We first deduce on-diagonal lower bounds. Indeed, letting $B_{1}:=B\left(x,\left(\lambda_{1} t\right)^{1 / \beta}\right)$ for some $\lambda_{1}>0$ to be specified later on, we see from (5.33) that

$$
\int_{B_{1}} p(t, x, y) \mathrm{d} \mu(y) \geqslant \int_{B_{1}} p_{B_{1}}(t, x, y) \mathrm{d} \mu(y) \geqslant c_{5}-c_{6} \lambda_{1}^{-1}=\frac{1}{2} c_{5}
$$

if $2 \lambda_{1}^{-1} c_{6}=c_{5}$. Thus, for all $x \in X$ and $0<t \leqslant r_{0}$, using Hölder's inequality,

$$
\begin{aligned}
p(2 t, x, x) & =\int_{X} p(t, x, y)^{2} \mathrm{~d} \mu(y) \\
& \geqslant \int_{B_{1}} p(t, x, y)^{2} \mathrm{~d} \mu(y) \\
& \geqslant \mu\left(B_{1}\right)^{-1}\left(\int_{B_{1}} p(t, x, y) \mathrm{d} \mu(y)\right)^{2} \\
& \geqslant c t^{-\alpha / \beta},
\end{aligned}
$$

giving an on-diagonal lower bound of $p(t, x, y)$. We next derive near-diagonal lower bounds of $p(t, x, y)$. Observe that, by (3.7) and (5.1),

$$
\mathcal{E}(p(t, x, \cdot)) \leqslant c t^{-(1+\alpha / \beta)}
$$

for all $x \in X$ and $0<t \leqslant r_{0}^{\beta}$. Therefore, by (5.61),

$$
\begin{aligned}
p(t, x, y) & \geqslant p(t, x, x)-|p(t, x, x)-p(t, x, y)| \\
& \geqslant c t^{-\alpha / \beta}-R(x, y)^{1 / 2} \mathcal{E}(p(t, x, \cdot))^{1 / 2} \\
& \geqslant c t^{-\alpha / \beta}-c_{9}\left(d(x, y)^{\beta-\alpha} t^{-(1+\alpha / \beta)}\right)^{1 / 2} \\
& \geqslant 2^{-1} c t^{-\alpha / \beta}
\end{aligned}
$$

if $d(x, y) \leqslant \varepsilon_{4} t^{1 / \beta}$ for a small $\varepsilon_{4}$ independent of $t, x$ and $y$. Finally, off-diagonal lower bounds of $p(t, x, y)$ follow from (5.61) and (5.62) by using the chain argument (see [2, pp. 36, 37] or [16, Corollary 3.5]). We omit the details. 
Acknowledgements. The author thanks K. J. Falconer for his comments, and Alexander Grigor'yan for pointing out the simpler proof of (3.7). The author also thanks the referee for his/her suggestions. This work was partly supported by NSFC Grant no. 10371062 and the Alexander von Humboldt Foundation.

\section{References}

1. D. G. Aronson, Bounds for the fundamental solution of a parabolic equation, Bull. Am. Math. Soc. 73 (1967), 890-896.

2. M. T. BARlow, Diffusions on fractals, in Lectures on Probability Theory and Statistics, Lecture Notes on Mathematics, Volume 1690, pp. 1-121 (Springer, 1998).

3. M. T. BARLOW AND R. F. BASs, Brownian motion and harmonic analysis on Sierpiński carpets, Can. J. Math. 51 (1999), 673-744.

4. M. T. Barlow And E. A. Perkins, Brownian motion on the Sierpiński gasket, Prob. Theory Relat. Fields 79 (1988), 543-623.

5. M. Barlow, T. Coulhon and T. Kumagai, Characterization of sub-Gaussian heat kernel estimates on strongly recurrent graphs, Commun. Pure Appl. Math. 58 (2005), 1642-1677.

6. E. B. DAviEs, Heat kernels and spectral theory (Cambridge University Press, 1989).

7. J. DodzIUK, Maximum principle for parabolic inequalities and the heat flow on open manifolds, Indiana Univ. Math. J. 32 (1983), 703-716.

8. E. B. FABES AND D. W. STROock, A new proof of Moser's parabolic Harnack inequality using the old ideas of Nash, Arch. Ration. Mech. Analysis 96 (1986), 327-338.

9. K. J. FALCONER, Fractal geometry: mathematical foundation and applications (Wiley, 1992).

10. W. FELLER, The general diffusion operator and positivity preserving semi-groups in one dimension, Ann. Math. (2) 60 (1954), 417-436.

11. P. J. Fitzsimmons, B. M. Hambly and T. Kumagai, Transition density estimates for Brownian motion on affine nested fractals, Commun. Math. Phys. 165 (1994), 595-620.

12. M. Fukushima, Y. Oshima And M. TAKeda, Dirichlet forms and symmetric Markov processes (de Gruyter, Berlin, 1994).

13. A. GRIGOR'YAN, Heat kernel upper bounds on fractal spaces, preprint (2005).

14. A. Grigor'YAn AND A. TElCs, Sub-Gaussian estimates of heat kernels on infinite graphs, Duke Math. J. 109 (2001), 451-510.

15. A. Grigor'yan And A. TelCs, Harnack inequalities and sub-Gaussian estimates for random walks, Math. Annln 324 (2002), 521-556.

16. A. GRIGOR'YAN, J. HU AND K.-S. LAU, Heat kernels on metric-measure spaces and an application to semilinear elliptic equations, Trans. Am. Math. Soc. 355 (2003), 2065-2095.

17. B. M. Hambly and T. Kumagai, Transition density estimates for diffusion processes on post critically finite self-similar fractals, Proc. Lond. Math. Soc. 78 (1999), 431-458.

18. J. KIGAMI, Analysis on fractals (Cambridge University Press, 2001).

19. J. Kigami, Harmonic analysis for resistance forms, J. Funct. Analysis 204 (2003), 399444 .

20. T. Kumagai, Heat kernel estimates and parabolic Harnack inequalities on graphs and resistance forms, Publ. RIMS Kyoto 40 (2004), 793-818.

21. D. RAY, Stationary Markov processes with continuous paths, Trans. Am. Math. Soc. 82 (1956), 452-493.

22. K. YOSIDA, Functional analysis (Springer, 1980). 